\title{
Age-dependent contribution of domain-general networks to
}

\section{semantic cognition}

Running title: Age-dependent contribution of domain-general networks

Sandra Martin ${ }^{1,2^{*}}$, Dorothee Saur ${ }^{2}$, Gesa Hartwigsen ${ }^{1}$

${ }^{1}$ Lise Meitner Research Group Cognition and Plasticity, Max Planck Institute for Human

Cognitive and Brain Sciences, Leipzig, Germany

${ }^{2}$ Language \& Aphasia Laboratory, Department of Neurology, University of Leipzig Medical

Center, Leipzig, Germany

* Corresponding author: Max Planck Institute for Human Cognitive and Brain Sciences,

Stephanstr. 1a, 04103 Leipzig, Germany. E-mail address: martin@cbs.mpg.de, phone: +49

34199402683. 


\begin{abstract}
Aging is characterized by a decline of cognitive control. In semantic cognition, this leads to the paradox that older adults usually show poorer task performance than young adults despite their greater semantic knowledge. So far, the underlying neural changes of these behavioral differences are poorly understood. In the current neuroimaging study, we investigated the contribution of domain-general networks to a verbal semantic fluency task in young and older adults. In both age groups, task processing was characterized by a strong positive integration within the multiple-demand as well as between the multiple-demand and the default mode system during semantic fluency. However, the behavioral relevance of strengthened connectivity differed between groups: While within-network functional connectivity in both networks predicted greater efficiency in semantic fluency in young adults, it was associated with a slower performance in older adults. Moreover, only young adults profited from increased connectivity between networks for their semantic memory performance. Our results suggest that the functional coupling of usually anti-correlated networks is critical for successful task processing, independent of age, when access to semantic memory is required. Furthermore, our findings lend novel support to the notion of reduced efficiency in the aging brain, due to neural dedifferentiation in semantic cognition.
\end{abstract}

Keywords: aging, connectivity, default mode network, multiple-demand network, language production 


\section{Introduction}

Semantic cognition is a fundamental human ability that is central to communication across the life span. Key facets of semantic cognition refer to our knowledge of the world and the meaning of words and sentences. With respect to cognitive changes across the adult life span, cognitive control processes - also referred to as fluid intelligence - are well established to steadily decline with increasing age (Hedden and Gabrieli, 2004) whereas semantic knowledge (so-called crystallized intelligence) has been shown to remain stable or might even increase with age due to the ongoing accrual of knowledge and experience across the life course (Verhaegen, 2003). In the domain of semantic cognition, the impact of aging thus seems to depend on both the specific cognitive demand of a task and the individual semantic knowledge.

At the neural level, cognitive changes with age are mirrored by large-scale reorganization processes at the structural and functional levels (Grady, 2012; Morcom and Johnson, 2015). Task-related performance changes in older adults have been associated with a pattern of dedifferentiation of neural activity (Park et al., 2004; Li et al., 2001) which is reflected by an under-recruitment of domain-specific regions (Lövdén et al., 2010) and reduced task-specific lateralization (Cabeza, 2002). Dedifferentiation is further characterized by an increased recruitment of areas in the domain-general multiple-demand network (MDN; Lövdén et al., 2010) and a reduced deactivation of regions in the default mode network (DMN; AndrewsHanna et al., 2007; Damoiseaux et al., 2008; Persson et al., 2007). A recent meta-analysis that investigated age-related effects on the neural substrates of semantic cognition confirmed the upregulation of the MDN in older adults for a variety of semantic tasks (Hoffman and Morcom, 2018).

In addition to local changes in task-related activity, alterations in the functional connectivity of large-scale neural networks have become a hallmark of brain aging 
(Damoiseaux et al., 2017; Li et al., 2015; Spreng et al., 2016). A common observation is that functional network segregation declines with age which is evident in the form of decreased within- and increased between-network functional connectivity (Chan et al., 2014; Geerligs et al., 2015; Spreng et al., 2016). These changes have been interpreted as dedifferentiation of network interactions, paralleling local task-related neural changes (Spreng and Turner, 2019). However, the majority of studies investigated these changes at rest and it is thus less clear how aging affects task-related functional connectivity and how this is associated with behavior.

The recently proposed default-executive coupling hypothesis of aging (DECHA; Turner and Spreng, 2015; Spreng and Turner, 2019) suggests that the observed activity increase in MDN regions and the reduced deactivation of the DMN co-occur and are functionally coupled in older adults. This shift in network coupling is based on the accrual of semantic knowledge and the parallel decline of cognitive control abilities. Older adults thus rely more strongly on their preserved semantic knowledge which is reflected by a reduced deactivation of DMN regions compared to young adults. According to this hypothesis, context and cognitive demand of a task determine if this increased default-executive coupling in older adults is beneficial or maladaptive. On this basis, the framework predicts a stable performance in older adults for tasks that rely on crystallized intelligence in the form of intrinsic prior knowledge and require little cognitive control whereas externally-directed cognitive tasks result in poorer performance in older adults due to their dependence on control resources.

So far, the integration of domain-general networks in semantic word retrieval in older adulthood is poorly understood. In this context, semantic fluency tasks, which require participants to generate words that belong to a specific category within a given time, provide a unique opportunity since they require an interaction of verbal semantic and general 
cognitive control processes (Gordon et al., 2018; Schmidt et al., 2017; Whiteside et al., 2016). Semantic fluency tasks test a natural and important communicative ability as they rely on accessing related concepts to retrieve words. Furthermore, semantic fluency is of high ecological validity, for example when writing a shopping list (Shao et al., 2014), and is frequently implemented as a measure of language and neuropsychological abilities in healthy as well as clinical populations (Schmidt et al., 2017). The impact of aging on semantic fluency is especially interesting since its strong link to semantic memory would predict preserved performance for older adults. Yet, the opposite pattern is usually observed, suggesting a high load on cognitive control processes for this task (e.g., Kavé and KnafoNoam, 2015; Gordon et al., 2018; Troyer et al., 1997). Most studies that implemented semantic fluency tasks in neuroimaging experiments reported age-related changes within domain-specific networks (Baciu et al., 2016; Marsolais et al., 2014) or pre-defined regions of interest, mainly in the prefrontal cortex (Meinzer et al., 2009; Meinzer et al., 2012a; Meinzer et al., 2012b). However, based on the outlined changes in semantic and cognitive control abilities, older adults could show a shift in network coupling with a stronger engagement of domain-general networks which might be additionally modulated by task demand.

Thus, the aim of the present study was to explore and compare the interplay of domain-specific and domain-general networks during word retrieval in healthy young and older adults. We implemented an fMRI study with a paced overt semantic fluency task, which included an explicit modulation of task difficulty. A counting task was used as a lowlevel verbal control task. First, we were interested in delineating the network for semantic fluency and its interaction with task demand. Secondly, we asked whether age modulates both activation patterns and behavioral performance. Finally, we were interested in taskrelated functional interactions between domain-specific and domain-general networks. To 
this end, we combined univariate whole-brain analyses with generalized psychophysiological interaction (gPPI) analyses. We applied traditional gPPI analyses to explore the functional coupling of the strongest activation peaks for semantic fluency. This allowed us to investigate the age-related contribution of domain-general networks to language processing. Furthermore, we used modified gPPI analyses to examine functional connectivity within and between regions of domain-general networks. We were interested in age-related effects on functional connectivity patterns and how within- and between-network functional connectivity relate to behavioral performance for both age groups. We expected a languagespecific left-lateralized network for semantic fluency. We further hypothesized that increased task demand (reflected by the contrast of semantic fluency with counting, as well as by the modulation of difficulty within the semantic fluency task) would affect task performance and should be accompanied by an increased recruitment of domain-general systems. With respect to task-related functional connectivity, we reasoned that older adults should demonstrate a stronger involvement of the DMN for the semantic fluency task based on their increased semantic knowledge. Moreover, a higher task load associated with general cognitive decline might further result in a stronger recruitment of the MDN in older adults. However, in line with neurocognitive theories of aging, the increased recruitment of domain-general systems might be associated with reduced specificity and efficiency; thus, overall leading to a poorer performance in the older adults.

\section{Materials and Methods}

\section{Participants}

Twenty-eight healthy older adults (mean age: 65.2 years, range: 60-69 years) and thirty healthy younger adults (mean age: 27.6 years, range: 21-34 years) completed our study. The data of 3 additional participants in the older group as well as individual runs of 6 participants 
were excluded due to excessive head movement during fMRI ( $>1$ voxel size). Groups were matched for gender. Participants in the younger group had significantly more years of education $(t(55.86)=5.2, p<.001)$. All participants were native German speakers and righthanded according to the Edinburgh Handedness Inventory (Oldfield, 1971). They had normal hearing, normal or corrected-to-normal vision, and no history of neurological or psychiatric conditions or contraindication to MR-scanning. Older adults were additionally screened for cognitive impairments using the Mini-Mental State Examination (Folstein et al., 1975; all $\geq 26 / 30$ points) and for depression with the Beck Depression Inventory (Beck et al., 1996; all $\leq 14$ / points). The study was approved by the local ethics committee of the University of Leipzig and conducted in accordance with the Declaration of Helsinki. Participants gave written informed consent prior to the experiment. They received 10 Euro per hour for their participation.

\section{Neuropsychological Assessment}

A battery of neuropsychological tests was administered to all participants to assess cognitive functioning. Verbal knowledge and executive language functions were measured with the German version of the spot-the-word test (Wortschatztest; Schmidt and Metzler, 1992; Baddeley et al., 1993), a German version of the reading span test (Daneman and Carpenter, 1980), and the semantic subtest of a verbal fluency test (Regensburger Wortflüssigkeitstest; Aschenbrenner et al., 2000). The latter comprised two 1-minute trials of semantic categories (surnames and hobbies) that were not part of the fMRI task. Additionally, executive functions were assessed with the Digit Symbol Substitution Test (Wechsler, 1944) and the Trail Making Test A/B (Reitan, 1958).

Group comparisons showed that older participants only performed better than the younger group on the spot-the-word test (Table 1 and Supplementary Fig. S1), which is considered a measure of lexical semantic knowledge and vocabulary. Consistent with our 
results, it has been shown to be robust to aging and cognitive decline (Baddeley et al., 1993; Law and O'Carroll, 1998; Cohen-Shikora and Balota, 2016). Our results confirm the maintenance of semantic memory across age (Grady, 2012) and an increase in size of vocabulary with age (Verhaegen, 2003). All other tests showed better performance for younger participants, which is in line with the assumption of a general decline in executive functions like working memory and processing speed with age (e.g., Balota et al., 2000; Zacks et al., 2000). However, when considering age-corrected norms, the older participants performed within normal ranges on all neuropsychological tests.

\section{Experimental Design}

All participants completed one fMRI session which was divided into two runs. Tasks consisted of a paced overt semantic fluency task and a control task of paced counting which were implemented in a block design in the scanner. We chose a paced design for our tasks since it has been shown to be less sensitive to motion artifacts and to yield robust brain activation patterns (Basho et al., 2007). Task blocks were $43 \mathrm{~s}$ long and separated by rest blocks of $16 \mathrm{~s}$ (Fig. 1A). Each block started with a $2 \mathrm{~s}$ visual word cue indicating whether participants were expected to generate category exemplars or count forward (1 to 9) or backward (9 to 1). This was followed by 9 consecutive trials of the same category or counting task, respectively. Trials within one block were separated by inter-stimulus intervals of 2-4 s. Participants were instructed to generate one exemplar for a category or one number per trial, which was indicated through a green cross on the screen, and to pause when the cross turned red (Fig. 1B and C). They were told not to repeat items and to say "next" if they could not think of an exemplar for the respective category. Each run contained 10 semantic fluency blocks, divided in easy and difficult categories, and 10 counting blocks, consisting of forward and backward counting, thus resulting in a total duration of 19.4 min per run. The order of blocks was counter-balanced and pseudo-randomized across participants. Before the fMRI 
experiment, participants received instructions and practiced the task with a separate set of categories outside the scanner. Stimuli were presented using the software Presentation (Neurobehavioral Systems, Berkeley, USA; version 18.0). Answers were recorded via a FOMRI III microphone (Optoacoustics, Yehuda, Israel).

Table 1. Demographic and neuropsychological characteristics of participants

\begin{tabular}{|c|c|c|}
\hline & $\begin{array}{l}\text { Young adults } \\
(n=30)\end{array}$ & $\begin{array}{l}\text { Older adults } \\
(n=28)\end{array}$ \\
\hline \multicolumn{3}{|l|}{ Demographics } \\
\hline Age (years) & $27.6(4.4)$ & $65.2(2.8)$ \\
\hline Gender (F:M) & $16: 14$ & $14: 14$ \\
\hline Education (years) & $18.7(2.6)$ & $15.2(2.5)^{*}$ \\
\hline Beck Depression Inventory (cut-off 18 points) & - & $4.7(4.1)$ \\
\hline \multicolumn{3}{|l|}{ Neuropsychological } \\
\hline Spot-the-word test (max. 40) & $29.1(3.2)$ & $31.5(2.5)^{*}$ \\
\hline Semantic fluency (sum surnames, hobbies) & $51.2(8.4)$ & $40.7(6.7)^{*}$ \\
\hline Reading span test (max. 6) & $3.5(1)$ & $2.9(0.7)^{*}$ \\
\hline Digit symbol substitution test (max. 90 in $90 \mathrm{~s}$ ) & $72.1(11.4)$ & $50.2(10.4)^{*}$ \\
\hline Trail Making Test A (time in s) & $17.3(5.8)$ & $25.4(6.4)^{*}$ \\
\hline Trail Making Test B (time in s) & $36.1(11.9)$ & $61.8(29.4)^{*}$ \\
\hline Mini-Mental State Examination (max. 30 points) & - & $28.36(1.2)$ \\
\hline
\end{tabular}

Note: Mean values of raw scores with standard deviations. * Significant differences between age groups at $p<0.01$.

\section{Stimuli}

Stimuli consisted of 20 semantic categories which were divided in 10 easy and 10 difficult categories. Difficulty was assessed in a separate pilot study with 24 older adults (10 male, mean age: 65 years, range: 60-69 years). Participants were recruited and screened using 
A Experimental paradigm

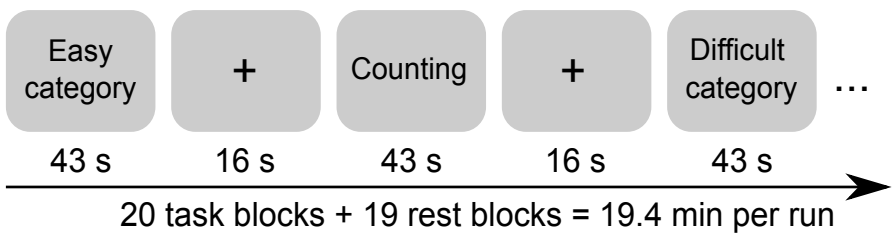

B Overt Semantic Word Generation Task

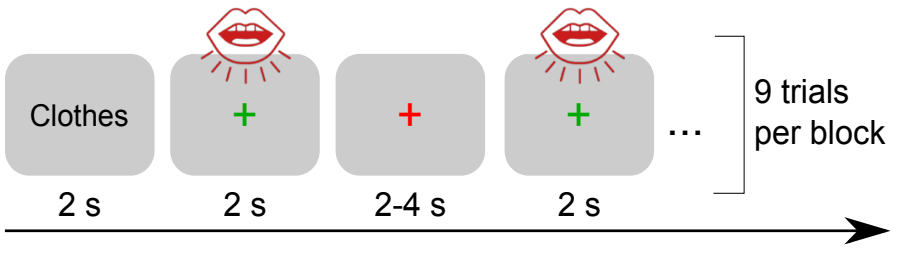

C Control task: Overt Counting

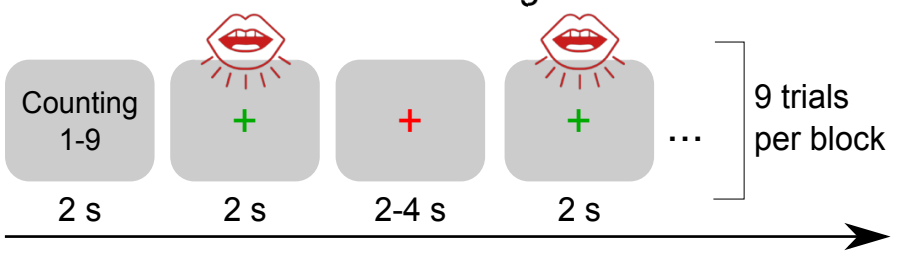

Figure 1. Experimental design. (A) fMRI experiment consisting of alternating blocks of a semantic fluency and a counting task separated by 16 -s rest periods. (B) and $(\mathrm{C})$ demonstrate the implementation of the paced design in both tasks. Procedures were identical for both tasks. Participants were instructed to produce one exemplar for a category or to say one number per green cross, respectively, and to pause when the cross turned red. Each block contained 9 trials which were separated by jittered inter-stimulus intervals. similar criteria as in the fMRI study.

They generated as many exemplars as

possible during 1-minute trials for 30

semantic categories which were taken

from German category-production

norm studies (Mannhaupt, 1983;

Glauer et al., 2007). Responses were recorded and subsequently transcribed and analyzed. Based on the total number of correct exemplars produced for each category, the 10 categories with the largest number of produced items (colors, body parts, clothing, types of sport, animals, car parts, professions, trees, food, and musical instruments) and the 10 categories with the fewest items (flowers, insects, metals, kitchen devices, tools, gardening tools, fishes, cosmetics, toys, and sweets) were chosen for the easy and difficult conditions of the semantic fluency task in the fMRI experiment, respectively. Easy $(M=16.8, S D=2.5)$ and difficult categories $(M=10.4, S D=2)$ differed significantly in the mean number of generated exemplars $(t(38.9)=9.2, p<0.001)$ during piloting.

\section{Data Acquisition and Preprocessing}

MR images were collected at a 3-Tesla Prisma Scanner (Siemens, Erlangen, Germany) with a 32-channel head coil. For the acquisition of fMRI data, a dual gradient echo-planar imaging multiband sequence (Feinberg et al., 2010) was used for optimal BOLD sensitivity across the 
whole brain (Poser et al., 2006; Halai et al., 2014). The following scanning parameters were applied: $\mathrm{TR}=2,000 \mathrm{~ms}$; $\mathrm{TE}=12 \mathrm{~ms}, 33 \mathrm{~ms}$; flip angle $=90^{\circ}$; voxel size $=2.5 \times 2.5 \times 2.5$ $\mathrm{mm}$ with an inter-slice gap of $0.25 \mathrm{~mm} ; \mathrm{FOV}=204 \mathrm{~mm}$; multiband acceleration factor $=2$. To increase coverage of anterior temporal lobe (ATL) regions, slices were tilted by $10^{\circ}$ of the AC-PC line. 616 images consisting of 60 axial slices in interleaved order covering the whole brain were continuously acquired per run. Additionally, field maps were obtained for later distortion correction $(\mathrm{TR}=8000 \mathrm{~ms} ; \mathrm{TE}=50 \mathrm{~ms})$. This study analyzed the data from echo 2 $(\mathrm{TE}=33 \mathrm{~ms})$ since preprocessing was performed using the software fMRIPrep (Esteban et al., 2019), which currently does not support the combination of images acquired at different echo times. We chose to use results from preprocessing with fMRIPrep since this pipeline provides state-of-the-art data processing while allowing for full transparency and reproducibility of the applied methods and a comprehensive quality assessment of each processing step which facilitates the identification of potential outliers. We also doublechecked results from preprocessing with fMRIPrep with a conventional SPM preprocessing pipeline of both echoes. The comparison of both pipelines did not reveal big differences in analysis results. A high-resolution, T1-weighted 3D volume was obtained from our in-house database (if it was not older than 2 years) or collected after the functional scans using an MPRAGE sequence (176 slices in sagittal orientation; TR $=2300 \mathrm{~ms}$; TE $=2.98 \mathrm{~ms}$; flip angle $=9^{\circ}$; voxel size $=1 \times 1 \times 1 \mathrm{~mm}$; no slice gap; FOV $=256 \mathrm{~mm}$ ).

Preprocessing was performed using fMRIPprep 1.2.6 (Esteban et al., 2019), which is based on Nipype 1.1.7 (Gorgolewski et al., 2017). In short, within the pipeline, anatomical images were processed using the software ANTs (Tustison et al., 2010) for bias field correction, skull stripping, coregistration, and normalization to the skull-stripped ICBM 152 Nonlinear Asymmetrical template version 2009c (Fonov et al. 2009). FreeSurfer (Dale, Fischl, and Sereno 1999) was used for brain surface reconstruction and FSL (Jenkinson et al. 
2012) for segmentation. Functional data of each run were skull stripped, distortion corrected, slice-time corrected, coregistered to the corresponding T1 weighted volume, and resampled to MNI152NLin2009cAsym standard space. Head-motion parameters with respect to the BOLD reference (transformation matrices, and six corresponding rotation and translation parameters) were estimated before any spatiotemporal filtering using FSL. For more details of the pipeline, see the section corresponding to workflows in fMRIPrep's documentation (https://fmriprep.org/en/1.2.6/workflows.html). After preprocessing, 29 volumes from the beginning of each run were discarded since they were collected for the combination of the short and long TE images via an estimation of the temporal signal-to-noise-ratio (Poser et al., 2006). This yielded 587 normalized images per run which were included in further analyses. The images were smoothed with a $5 \mathrm{~mm}^{3}$ FWHM Gaussian kernel using Statistical Parametrical Mapping software (SPM12; Wellcome Trust Centre for Neuroimaging), implemented in MATLAB (version 9.3/2017b).

\section{Data Analysis}

\section{Behavioral data}

Response recordings during the semantic fluency task were cleaned from scanner noise using Audacity (version 2.3.2, https://www.audacityteam.org/) and verbal answers and onset times were transcribed by 3 independent raters. Repetitions of words within a category were counted as incorrect, incomplete answers and null reactions were marked separately, and full categories that had been missed by participants (in total 10 categories) were excluded from the analyses. Statistical analyses were performed with R via RStudio (R Core Team, 2018) and the packages lme4 (Bates et al., 2015) for mixed models and ggplot2 (Wickham, 2016) for visualizations. We used sum coding (ANOVA-style encoding) for all categorical predictors. In this way, the intercept represents the mean across conditions (grand mean), and the model coefficients represent the difference between the grand mean and the mean of the 
respective condition. For the analysis of accuracy, a generalized linear mixed-effects logistic regression was used accounting for the binary nature of the response variable (Table 2, Equation 1). For response time, a linear mixed-effects model was fit to the log-transformed data (Table 2, Equation 2). As fixed effects, we entered age, condition, and difficulty into the models. As random effects, we had intercepts for participants and categories. P-values were obtained by likelihood ratio tests of the full model with the effect in question against the model without the effect in question. Post-hoc comparisons were applied using the package emmeans (Lenth et al., 2020).

Table 2. Equations of regression models

$$
\text { Accuracy } \sim \text { Age + Condition + Difficulty }+ \text { Age * Condition + Age * Difficulty + }
$$

$$
(1 \mid \text { Subject })+(1 \mid \text { Category })
$$

(2)

$$
\text { Log }(\text { Response time }) \sim \text { Age }+ \text { Condition + Difficulty }+ \text { Age * Condition + Age * }
$$

$$
\text { Difficulty }+(1 \mid \text { Subject })+(1 \mid \text { Category })
$$

(3)

$$
\text { Beta weight } \sim \text { Network }+ \text { Age }+ \text { Condition }+ \text { Network * Age }+ \text { Network * Condition }+
$$

$$
\text { Age * Condition }+(1 \mid \text { Subject })
$$
(4)

$$
\mathrm{PPI}_{\text {contrast }} \sim 1
$$

(5)

$$
\mathrm{PPI}_{\text {network }} \sim \text { Age }
$$

(6)

$$
\text { Accuracy } \sim \mathrm{PPI}_{\mathrm{MDN}}+\mathrm{PPI}_{\mathrm{DMN}}+\mathrm{PPI}_{\mathrm{MDN} \_D M N}+\mathrm{Age}+\left(\mathrm{PPI}_{\mathrm{MDN}}+\mathrm{PPI}_{\mathrm{DMN}}+\mathrm{PPI}_{\mathrm{MDN} \_D M N}\right) \text { * }
$$

$$
\text { Age }+(1 \mid \text { Subject })+(1 \mid \text { Category })
$$

$$
\log (\text { Response time }) \sim \mathrm{PPI}_{\mathrm{MDN}}+\mathrm{PPI}_{\mathrm{DMN}}+\mathrm{PPI}_{\mathrm{MDN} \_ \text {DMN }}+\mathrm{Age}+\left(\mathrm{PPI}_{\mathrm{MDN}}+\mathrm{PPI}_{\mathrm{DMN}}+\right.
$$

$$
\left.\mathrm{PPI}_{\text {MDN_DMN }}\right){ }^{*} \text { Age }+(1 \mid \text { Subject })+(1 \mid \text { Category })
$$




\section{Functional MRI data}

Functional MRI data were modelled in SPM using the two-level approach. On the first level, a general linear model (GLM) was implemented for each participant. The GLM included regressors for the task blocks of the 4 experimental conditions (easy categories, difficult categories, counting forward, and counting backward) and nuisance regressors consisting of the six motion parameters and individual regressors for strong volume-to-volume movement as indicated by values of framewise displacement $>0.9$ (Siegel et al., 2014). A two-sample $t$ test indicated that there was no significant difference between older adults $(M=15.67, S D=$ $20.04)$ and young adults $(M=7.5, S D=8.35)$ with respect to the number of regressed volumes $(t(28.76)=1.66, p=0.11)$. Additionally, an individual regressor of no interest was included in the design matrix if a participant had missed a whole task block during the experiment $(n=10)$. Before model estimation, a high-pass filter with a cut-off at $128 \mathrm{~s}$ was applied to the data. Statistical parametric maps were generated by estimating the contrast for each condition against rest as well as the direct contrasts between conditions. At the second level, contrast images were entered into a random effects model. For each participant, an averaged mean-centered value of response time was entered as covariate of no interest in the design matrix. For within-group comparisons, one-sample $t$-tests were calculated for the main task-related contrasts Semantic fluency $>$ Counting and Counting $>$ Semantic fluency. To evaluate the modulation of task difficulty within the semantic fluency task, the contrasts Easy $>$ Difficult categories and Difficult $>$ Easy categories were computed.

To investigate the effect of age on task-related activity, we conducted between-group

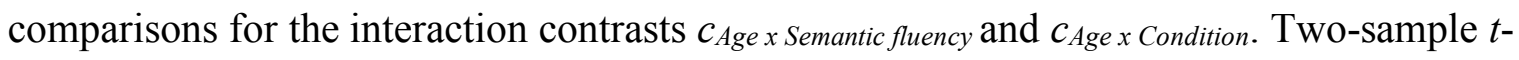
tests were carried out using the individual contrast images from the first-level analysis. To ensure that potential areas were indeed active in the respective group, all interactions were characterized by inclusively masking each contrast with significant voxels of the minuend (at 
$p<0.001$, uncorr., cf. Noppeney et al., 2006, Meinzer et al., 2012b). A gray matter mask which restricted statistical tests to voxels with a gray matter probability $>0.3$ (SPM12 tissue probability map) was applied to all second-level analyses. All results except for the interaction contrasts were corrected for multiple comparisons applying a peak level threshold at $p<0.05$ with the family-wise error (FWE) method and a cluster-extent threshold of 20 voxels. Interaction results were thresholded at $p<0.05$ at the cluster level with FWE correction and a voxel-wise threshold at $p<0.001$. Anatomical locations were identified with the SPM Anatomy Toolbox (Eickhoff et al., 2005, version 2.2c) and the Harvard-Oxford cortical structural atlases distributed with FSL (https://fsl.fmrib.ox.ac.uk). Brain results were rendered by means of BrainNet Viewer (Xia et al., 2013, version 1.7) and MRIcroGL (https://www.mccauslandcenter.sc.edu/mricrogl/, version 1.2.20200331).

Since the strongest activation peaks for both tasks were found in the domain-general systems MDN and DMN, we decided to examine the amount of activation for each condition and age group. We applied binary masks of both networks to analyses within the group of young adults to identify clusters that fell within the respective network. By basing our analysis on activity in the young adults, we ensured that more complex analyses were based on the same number of regions in both age groups. Further, this allowed us to investigate agerelated differences in these regions knowing that they are relevant for task processing in young adults. The MDN mask was based on the anatomical parcels of the MD system defined by Fedorenko et al. (2013; available at https://evlab.mit.edu/funcloc/). For the DMN, a mask was created from the 7 networks parcellation by Schaefer et al. (2018). For the contrast Semantic fluency $>$ Counting, peak global and local maxima were found in the MDN, whereas the reverse contrast identified clusters that are typically associated with the DMN. Due to the small number $(n=3)$ of peak clusters for the contrast Counting $>$ Semantic fluency with FWE correction at peak-level, we decided to apply a more lenient threshold 
(FWE-corrected at cluster-level, $p<0.001$ at peak-level) for the identification of regions associated with the DMN. This allowed us to extract a similar number of peak maxima for the MDN and the DMN, and provided a much more representative picture of the DMN as a whole. In total, we identified 14 peak maxima in the MDN, and 17 peak maxima in the DMN, respectively (Table 3). Regions of interest (ROIs) for these maxima were created using the MarsBar toolbox (Brett et al., 2002; version 0.44). To this end, identified clusters were extracted from contrast images, spheres of $5 \mathrm{~mm}$ from each maxima coordinate were created, and, in a last step, both images were combined. Subsequently, we extracted parameter estimates for these ROIs from the individual contrast images for Semantic fluency $>$ Rest and Counting $>$ Rest. The data were then entered into a linear mixed-effects model with network, age, and condition as fixed effects. A random intercept was included for participants (Table 2, Equation 3). Categorical predictors were sum coded. Significance values were obtained through likelihood ratio tests using the package lme4 (Bates et al., 2015). Post-hoc comparisons were applied using the package emmeans (Lenth et al., 2020).

\section{Connectivity analyses}

We conducted psychophysiological interaction (PPI) analyses using the gPPI toolbox for SPM12 (McLaren et al., 2012) to investigate task-related modulation of functional connectivity associated with the semantic fluency and the counting task. Furthermore, we applied a modified version of gPPI methods to examine functional connections between individual ROIs during the semantic fluency task (Pongpipat et al., 2020). Seed regions were defined for all previously identified global maxima that were located within the MDN and the DMN (Table 3). For each participant, ROIs were created by searching for the individual peaks within a bounding region of $10 \mathrm{~mm}$ relative to the group peak and then drawing a sphere mask ( $5 \mathrm{~mm}$ in diameter) around the individual peak of a given contrast at a threshold of $p<0.01$. Regression models were set up for each ROI in each participant containing the 
Table 3. Regions of interest within domain-general networks.

\begin{tabular}{|c|c|c|c|c|c|}
\hline ROI & Hemi & $x$ & $y$ & $\mathbf{z}$ & Region \\
\hline \multicolumn{6}{|c|}{ MDN (from contrast Semantic fluency > Counting) } \\
\hline 1 & $\mathrm{~L}$ & -31 & 25 & 2 & Insula \\
\hline 2 & $\mathrm{~L}$ & -4 & 25 & 40 & preSMA \\
\hline 3 & L & -6 & 12 & 51 & preSMA \\
\hline 4 & $\mathrm{R}$ & 13 & 27 & 29 & $\mathrm{dACC}$ \\
\hline 5 & L & 4 & 20 & 40 & $\mathrm{dACC}$ \\
\hline 6 & L & -4 & 2 & 29 & $\mathrm{dACC}$ \\
\hline 7 & $\mathrm{R}$ & 31 & 27 & 2 & Insula \\
\hline 8 & $\mathrm{R}$ & 38 & 20 & -4 & Insula \\
\hline 9 & L & -29 & -65 & 51 & SPL \\
\hline 10 & L & -29 & -72 & 43 & $A G$ \\
\hline 11 & L & -34 & -57 & 40 & IPL \\
\hline 12 & $\mathrm{R}$ & 36 & 42 & 32 & MFG \\
\hline 13 & $\mathrm{R}$ & 31 & 55 & 26 & MFG \\
\hline 14 & $\mathrm{R}$ & 33 & 37 & 21 & MFG \\
\hline \multicolumn{6}{|c|}{ DMN (from contrast Counting > Semantic fluency) } \\
\hline 15 & $\mathrm{R}$ & 51 & 10 & -31 & TP \\
\hline 16 & $\mathrm{R}$ & 48 & -10 & -15 & STG \\
\hline 17 & $\mathrm{R}$ & 8 & -65 & 29 & Precuneus \\
\hline 18 & $\mathrm{R}$ & 11 & -52 & 35 & Precuneus \\
\hline 19 & L & -9 & -52 & 35 & Precuneus \\
\hline 20 & L & -56 & 2 & -20 & MTG \\
\hline 21 & L & -54 & 10 & -31 & TP \\
\hline 22 & $\mathrm{~L}$ & -6 & 27 & -6 & ACC \\
\hline
\end{tabular}

deconvolved time series of the

first eigenvariate of the BOLD

signal from the respective ROI

as the physiological variable,

the four task conditions

convolved with the HRF as the

psychological variable, and the

interaction of both variables as

the PPI term. Subsequently,

first-level GLMs were

calculated. For the gPPI proper

methods, contrast images were

then entered into a random

effects model for group

analyses in SPM. We restricted

this analysis to the strongest

peaks of both contrasts

(Semantic fluency $>$ Counting

and Counting $>$ Semantic

fluency) that fell within the

MDN and DMN, respectively.

This included the left pre-SMA,

bilateral insulae, the right

temporal pole and the right

precuneus. Our main contrast of 


\begin{tabular}{llllll}
\hline 23 & $\mathrm{~L}$ & -6 & 42 & -4 & ACC \\
24 & $\mathrm{~L}$ & -54 & -62 & 35 & AG \\
25 & $\mathrm{~L}$ & -41 & -60 & 26 & AG \\
26 & $\mathrm{~L}$ & -46 & -62 & 18 & $\mathrm{MTG}$ \\
27 & $\mathrm{~L}$ & -46 & -75 & 35 & $\mathrm{AG}$ \\
28 & $\mathrm{~L}$ & -49 & -67 & 43 & $\mathrm{AG}$ \\
29 & $\mathrm{R}$ & 51 & -57 & 26 & $\mathrm{AG}$ \\
30 & $\mathrm{R}$ & 46 & -65 & 48 & AG \\
31 & $\mathrm{R}$ & 43 & -72 & 35 & AG \\
\hline
\end{tabular}

Note: Coordinates are given in MNI standard space. Abbreviations: Hemi Hemisphere; Pre-SMA Presupplementary motor area; MFG/MFGd/MFGv Middle frontal gyrus dorsal/ventral; SPL Superior parietal lobe; IntraCAL Intracalcarine gyrus; TP Temporal pole; dACC Dorsal anterior cingulate cortex; MTG Middle temporal gyrus; MDN Multiple-demand network; DMN Default mode network. interest Semantic fluency $>$

Counting was examined in

within-group as well as

between-group comparisons by

conducting one-sample $t$-tests

and a two-sample $t$-test,

respectively. Multiple

comparison correction was

performed with the FWE

method at $p<0.05$ at peak level

and a cluster-extent value of 20

voxels. A gray matter mask was

applied to all group analyses as

described for the task-based fMRI data analysis.

For the modified gPPI, we used the individual first-level GLMs to retrieve parameter estimates (mean regression coefficients). Estimates were extracted for the PPI variable Semantic fluency $>$ Counting for each seed-to-target ROI (1 regression coefficient [PPI] * 31 seed ROIs * 30 target ROIs $=930$ parameter estimates per participant). Subsequent group analyses were performed in RStudio (R Core Team, 2018) with the package lme4 (Bates et al., 2015), and visualized using the ggplot2 (Wickham, 2016) and the ggeffects (Lüdecke, 2018) packages. Categorical predictors were sum coded. We were interested in the functional connectivity within and between MDN and DMN regions for each age group. To this end, we calculated intercept-only GLMs where each parameter estimate of each seed and target combination was entered into the model except when seed and target were identical (Table 2, equation 4). The $\alpha$-level (Type I error) for post-hoc comparisons was adjusted using the Meff 
correction (Derringer, 2018). This method estimates the effective number of tests (Meff) from the correlations among tested variables and thereby allows for adjusting statistical significance thresholds for multiple comparisons without assuming independence of all tests (Derringer, 2018). The Meff value for MDN and MDN variables was calculated to be 27.5. By dividing this value by the overall $\alpha$ of 0.05 , we obtained a Meff-corrected $\alpha$ of 0.0018 . For subsequent analyses, the individual parameter estimates of each seed-to-target combination were averaged to create one value per participant for within-MDN, withinDMN, and between-network functional connectivity (3 parameter estimates per participant). To test for an effect of age group on functional connectivity, the parameter estimates were then entered into a GLM with age group as independent variable (Table 2, equation 5). We used Meff-correction to adjust for multiple comparisons. A Meff value of 2.49 yielded a Meff-corrected $\alpha$ of 0.02 .

Furthermore, we were interested in the effect of within- and between-network functional connectivity on participants' behavioral performance during the in-scanner semantic fluency task. To this end, we calculated generalized mixed-effects logistic regressions for the accuracy data (Table 2, equation 6) and linear mixed-effects models for the log-transformed response time data (Table 2, equation 7). The mean-centered PPI $\mathrm{I}_{\text {network }}$ variables and age group as well as their interaction terms were entered as fixed effects. Random intercepts were included for participants and semantic categories.

Finally, to assess how the observed changes in network properties were related to cognitive performance and semantic memory in general, we performed correlation analyses with the neuropsychological measures that had been tested outside of the scanner. Due to the collinearity of some neuropsychological tests, we first performed a factor analysis on the standardized test scores using maximum likelihood estimation and varimax rotation in RStudio with the package stats (R Core Team, 2018). Based on the hypothesis test $\left(\chi^{2}=\right.$ 
$14.04, p=0.081)$ two factors with an eigenvalue $>1$ were chosen. For subsequent correlations with functional connectivity measures participant factor scores extracted via regression methods were used.

\section{Results}

\section{Behavioral Results}

For response accuracy, we fitted a generalized linear mixed-effects model for a binomial distribution. Likelihood ratio tests indicated significant main effects of condition $\left(\chi^{2}=21.55\right.$, $p<0.001)$ and task difficulty $\left(\chi^{2}=27.43, p<0.001\right)$ but not of age group $\left(\chi^{2}=0.19, p=\right.$ 0.66). Further, we detected a significant two-way interaction between age and difficulty $\left(\chi^{2}=\right.$ 9.76, $p=0.002)$ and condition and difficulty $\left(\chi^{2}=3.89, p=0.049\right)$ as well as a significant three-way interaction between age, condition, and difficulty $\left(\chi^{2}=9.28, p=0.002\right)$. Post-hoc tests applying Bonferroni-corrected pairwise comparisons showed that both age groups produced more correct items in the counting than in the semantic fluency task (all $p<0.001$ ) and more items for the easy than the difficult semantic categories (all $p<0.001$; Fig. 2A; Supplementary Tables S1 and S2).

Response times were analyzed fitting a linear mixed-effects model after logtransformation of the data. Likelihood ratio tests revealed main effects of age group $\left(\chi^{2}=\right.$ $17.23, p<0.001)$, condition $\left(\chi^{2}=21.40, p<0.001\right)$, and difficulty $\left(\chi^{2}=21.02, p<0.001\right)$.

There was a significant interaction between age and condition $\left(\chi^{2}=69.46, p<0.001\right)$. Posthoc tests using Bonferroni-corrected pairwise comparisons showed that both age groups responded significantly slower during the semantic fluency task than the counting task and during the difficult than the easy condition (all $p<0.001$ ). Furthermore, young adults responded generally faster than older adults during the semantic fluency task $(p<0.001)$, 
A

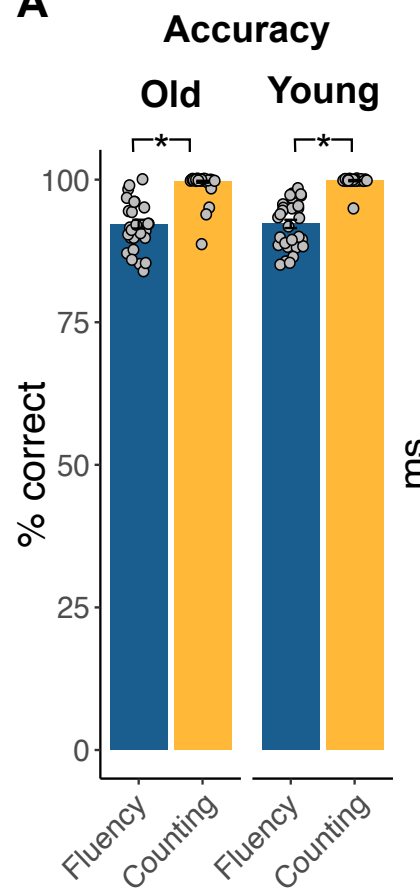

\section{Mean RT}

Old Young

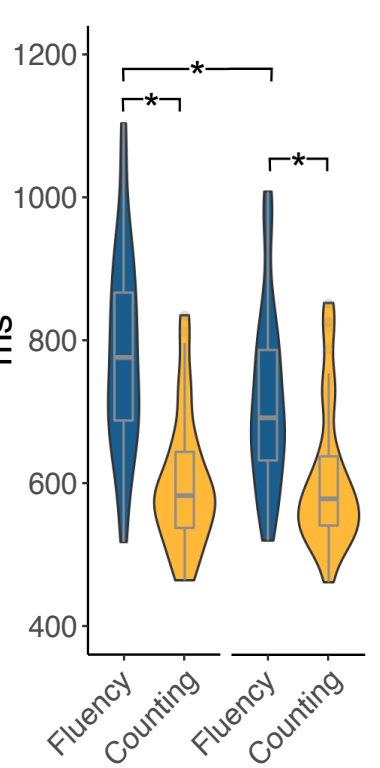

B

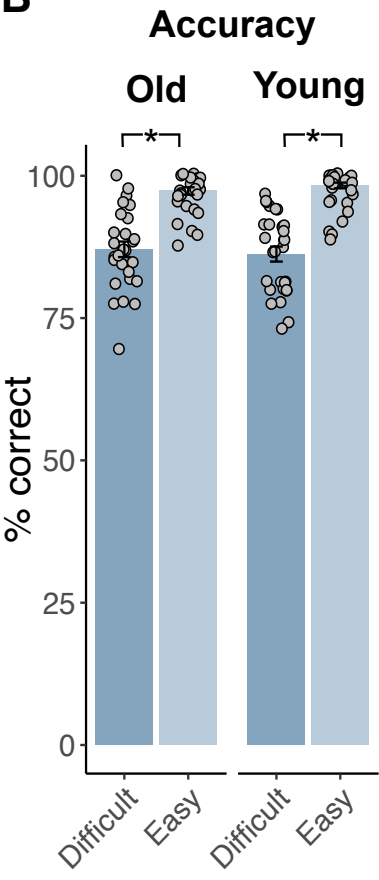

Mean RT

Old Young

Figure 2. Behavioral results for both age groups. Bar graphs overlaid with mean individual data points for accuracy and violin plots with box plots for mean response times for (A) both tasks (semantic fluency and counting) and (B) difficulty levels (easy and difficult) within semantic fluency. Old: Older adults, Young: young adults. $* p<0.001$ (Bonferroni-corrected for pairwise comparisons).

independent of the level of difficulty, but not during the counting task ( $p=0.05$; Fig. 2B;

Supplementary Tables S1 and S3).

\section{Functional MRI Data}

\section{The effect of task within groups}

Both age groups showed similar activation patterns for the main effects of our tasks compared to rest. For semantic fluency, we found a left-lateralized fronto-temporo-parietal network with additional clusters in right frontal and temporal areas, bilateral caudate nuclei, and the cerebellum (Supplementary Fig. S2; Supplementary Tables S4 and S5). The main effect of the less demanding task counting was evident in bilateral activation of sensorimotor cortices and the cerebellum (Supplementary Fig. S2; Supplementary Tables S6 and S7).

Within each age group, we were interested in the difference in brain activation between the 

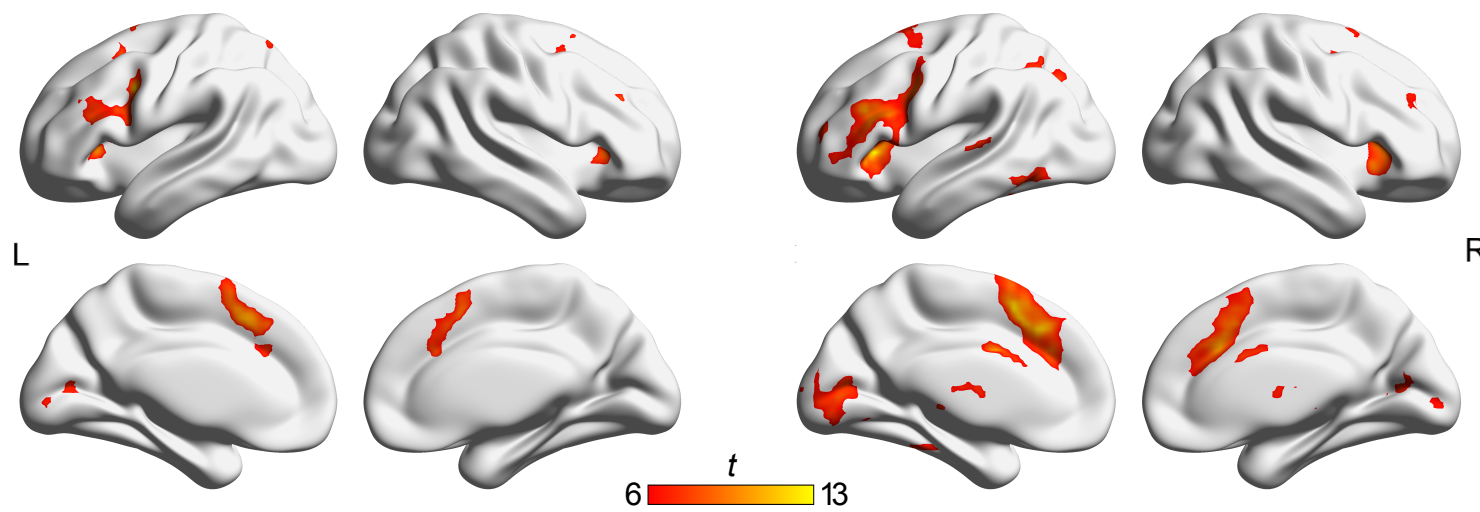

B Counting > Semantic fluency

Older adults

Young adults
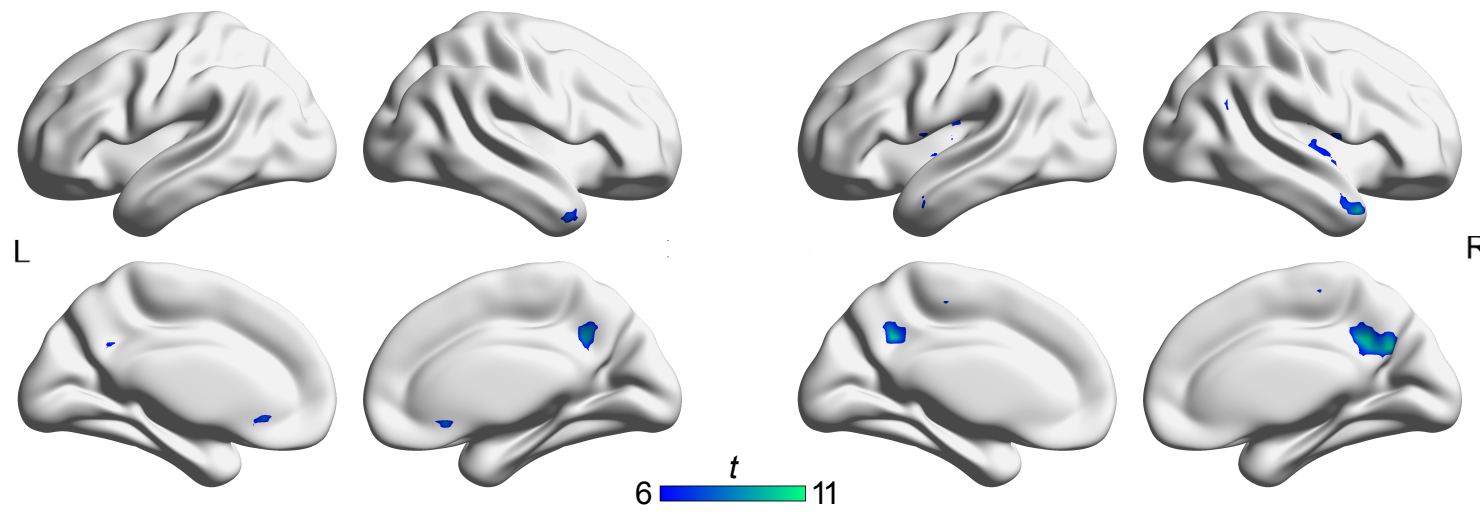

C

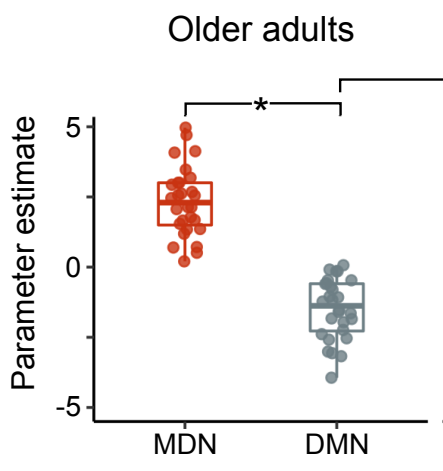

Semantic fluency $>$ Rest Young adults

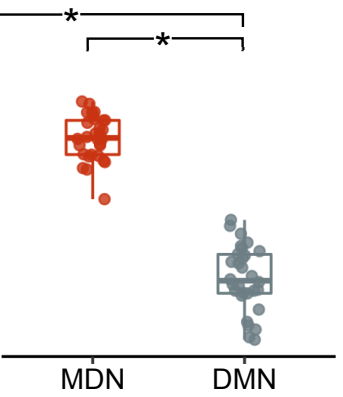

Older adults

Counting > Rest
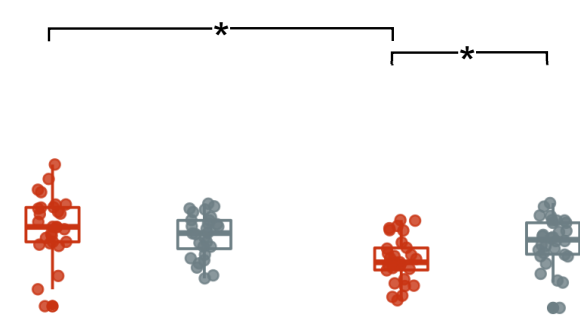

MDN DMN

Figure 3. Functional MRI results from univariate analyses for each age group and parameter estimates for peak maxima identified within the MDN and the DMN. (A \& B) Results are FWEcorrected at $p<0.05$ at peak level with a minimum cluster size $=20$ voxel. Unthresholded statistical maps are available at https://neurovault.org/collections/9072/. (C) * Significant effects are Bonferroni-corrected. Abbreviations: MDN Multiple-demand network; DMN Default mode network. 
more demanding semantic fluency task and the automatic speech counting task as well as in the impact of the modulation of task difficulty in the semantic fluency task. For the older adults, the contrast Semantic fluency $>$ Counting revealed a bilateral frontal network with its strongest activation peaks in middle frontal gyri, bilateral insulae extending into inferior frontal gyri, and midline structures comprising superior and medial frontal gyri. Activation in the left hemisphere was further observed in the angular gyrus and superior parietal lobe. Additional bilateral activation peaks were found in the cerebellum, caudate nuclei, calcarine gyri, and thalami (Fig. 3A; Supplementary Table S8). Younger adults demonstrated a similar pattern of activation for the contrast Semantic fluency $>$ Counting, albeit with generally larger clusters in the frontal network (Fig. 3A; Supplementary Table S9). Analyses further yielded separate clusters in the dorsal anterior cingulate cortex (dACC) and the left superior temporal gyrus for the younger group which were not present in the older participants. The reverse contrast (Counting $>$ Semantic fluency) revealed stronger activation in the right hemisphere for both groups. Results showed clusters in the right temporal pole and bilateral precunei (Fig. 3B; Supplementary Tables S10 and S11). In the younger group, additional clusters were observed in bilateral insulae and the middle temporal gyrus. When we applied a more lenient threshold of $p<0.001$ at peak-level and FWE-correction $(p<0.05)$ at clusterlevel, additional peaks were observed in bilateral parietal lobes including angular gyri and the anterior cingulate cortex (Supplementary Table S12).

A linear mixed-effects model was fit for the mean value of parameter estimates for all peak clusters in the MDN and DMN (Table 3), respectively. Likelihood ratio tests indicated significant effects for the explanatory variables network $\left(\chi^{2}=92.73, p<0.001\right)$, age $\left(\chi^{2}=\right.$ 11.03, $p=0.017)$, and condition $\left(\chi^{2}=23.04, p<0.001\right)$. We further found a significant interaction between network and condition $\left(\chi^{2}=196.14, p<0.001\right)$ and a significant threeway interaction between network, age, and condition $\left(\chi^{2}=18.58, p<0.001\right)$. Post-hoc 
comparisons applying Bonferroni correction revealed an effect of age for the DMN for the Contrast Semantic fluency $>$ Rest with older adults showing stronger activity in DM regions than young adults $(t=4.89, p<0.001)$ as well as an effect of age for the MDN for the Contrast Counting $>$ Rest with older adults showing stronger activity in MD regions than young adults $(t=4.81, p<0.001)$. Moreover, post-hoc tests showed that, in general, the MDN was activated for the semantic fluency task across age groups whereas the DMN showed deactivation $(t=24.99, p<0.001)$. For the counting task, there was no difference in activation between both networks $(t=0.81, p=0.42$; Fig. 3C; Supplementary Tables S13 and S14).

\section{The effect of task difficulty within groups}

To investigate the effect of task difficulty on functional brain activation, we contrasted easy and difficult categories from the semantic fluency task in both age groups. We found a significant result only for young adults for the contrast Easy $>$ Difficult categories in the right middle frontal gyrus (Supplementary Table S15).

\section{Between-group comparisons}

We were interested in the effect of age on task-related activations. For the interaction of both tasks compared to baseline, we found a group difference only during the semantic fluency task for older adults. We detected stronger activity in right frontal regions including superior frontal gyrus and inferior frontal gyrus as well as bilateral parietal lobes (Fig. 4A; Table 4). We were further interested in the interaction of age and condition. The contrast Semantic fluency $>$ Counting revealed a significant interaction with age only for young adults. Stronger activity was observed in the paracingulate gyrus, pre-SMA, and the dACC (Fig. 4B; Table 4). The interaction of age with task difficulty (easy and difficult semantic categories) did not yield any significant results. 
A Older adults $>$ Young adults Semantic fluency $>$ Rest

$x-36$

$\times 30$

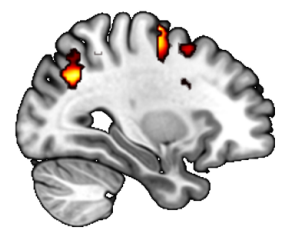

3.2 $t$

6.8

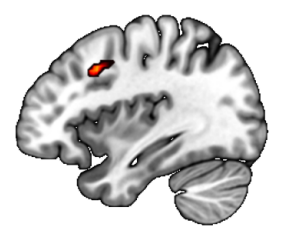

B Young adults $>$ Older adults

Semantic fluency $>$ Counting

$x 51$
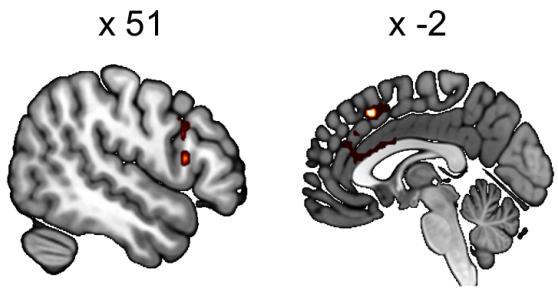

$\mathrm{x} 1$

$x 8$

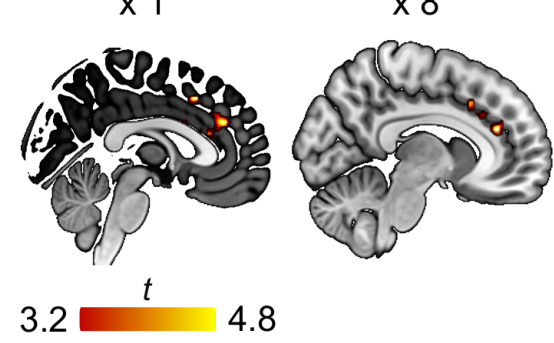

$3.2 \square-4.8$

Figure 4. Functional MRI results for interaction effects. Cluster corrected at FWE $p<0.05$ with a voxel-wise threshold at $p<0.001$. (A) Restricted to voxels that showed a significant effect of semantic fluency in older adults and (B) restricted to voxels that showed a significant effect of semantic fluency in young adults. Statistical maps are available at https://neurovault.org/collections/9072/.

Table 4. Results for age-dependent differences in task-related activity.

\begin{tabular}{lcccccc}
\hline Anatomical structure & Hemi & $k$ & $t$ & $x$ & $y$ & $z$ \\
\hline Interaction older $>$ young adults for & & & & & & \\
\hline
\end{tabular}

[older adults: semantic fluency $>$ rest])

\begin{tabular}{|c|c|c|c|c|c|c|}
\hline Superior frontal gyrus & $\mathbf{R}$ & 110 & 6.84 & 28 & -8 & 65 \\
\hline Precentral gyrus & $\mathrm{R}$ & & 5.53 & 28 & -8 & 54 \\
\hline Superior parietal lobe & $\mathbf{R}$ & 263 & 6.82 & 18 & -60 & 60 \\
\hline Middle occipital gyrus & $\mathrm{R}$ & & 6.04 & 31 & -62 & 35 \\
\hline Precuneus & $\mathrm{R}$ & & 5.93 & 11 & -55 & 60 \\
\hline Precuneus & $\mathrm{R}$ & & 5.39 & 13 & -67 & 62 \\
\hline Middle frontal gyrus & $\mathbf{L}$ & 74 & 5.38 & -36 & 15 & 38 \\
\hline Middle frontal gyrus & $\mathrm{L}$ & & 4.05 & -46 & 17 & 38 \\
\hline Precentral gyrus & $\mathrm{L}$ & & 3.76 & -41 & 2 & 48 \\
\hline Inferior parietal lobe & $\mathbf{L}$ & 109 & 5.12 & -31 & -45 & 54 \\
\hline Superior parietal lobe & $\mathrm{L}$ & & 4.32 & -19 & -60 & 46 \\
\hline Precuneus & $\mathrm{L}$ & & 4.28 & -11 & -70 & 48 \\
\hline Superior parietal lobe & $\mathrm{L}$ & & 4.19 & -16 & -62 & 60 \\
\hline Middle frontal gyrus & $\mathbf{R}$ & 132 & 4.96 & 36 & 5 & 38 \\
\hline
\end{tabular}




\begin{tabular}{|c|c|c|c|c|c|}
\hline Inferior frontal gyrus, p.tr. & $\mathrm{R}$ & & 4.53 & 36 & 17 \\
\hline Inferior frontal gyrus, p.op. & $\mathrm{R}$ & & 4.07 & 48 & 17 \\
\hline Inferior frontal gyrus, p.op. & $\mathrm{R}$ & & 3.73 & 51 & 15 \\
\hline Middle frontal gyrus & $\mathbf{R}$ & 74 & 4.47 & 26 & 12 \\
\hline
\end{tabular}

Interaction young > older adults for semantic fluency > counting (inclusively masked with [young adults: semantic fluency > counting])

\begin{tabular}{lllllll}
\hline Pre-SMA & L & 79 & $\mathbf{4 . 8 4}$ & $-\mathbf{1}$ & $\mathbf{2 0}$ & $\mathbf{4 6}$ \\
Pre-SMA & $\mathrm{R}$ & & 3.81 & 11 & 27 & 32 \\
Pre-SMA & $\mathrm{L}$ & & 3.55 & -1 & 10 & 48 \\
Anterior cingulate cortex & $\mathrm{R}$ & $\mathbf{1 1 4}$ & $\mathbf{4 . 5 8}$ & $\mathbf{8}$ & $\mathbf{3 7}$ & $\mathbf{2 1}$ \\
Pre-SMA & $\mathrm{R}$ & & 4.36 & 1 & 37 & 26 \\
Anterior cingulate cortex & $\mathrm{L}$ & & 4.13 & -1 & 20 & 21 \\
Anterior cingulate cortex & $\mathrm{L}$ & & 3.58 & -4 & 5 & 29 \\
\hline
\end{tabular}

Note: Cluster corrected at FWE $p<0.05$ with a voxel-wise threshold at $p<0.001$. Coordinates are given in MNI standard space, cluster size $(\mathrm{k})$ is given in $\mathrm{mm}^{3}$. Note that no significant differences above cluster correction threshold were found for (i) interaction young $>$ older adults for semantic fluency > rest, (ii) interaction older $>$ young adults for semantic fluency $>$ counting. Abbreviations: Hemi Hemisphere; p. tr. pars triangularis; p.op. pars opercularis; pre-SMA pre-supplementary motor area.

\section{Generalized psychophysiological interactions}

Based on the activation patterns from our univariate within-group analyses, we conducted traditional gPPI analyses for the 5 strongest activation peaks that fell within the MDN or DMN. We asked whether and how increased semantic task demands modulate connectivity of our ROIs.

\section{Whole-brain functional connectivity for semantic fluency}

Three ROIs were extracted from the univariate contrast Semantic fluency $>$ Counting, the left pre-SMA and bilateral insulae. For the seeds in the left pre-SMA and left insula, analyses 
revealed only significant clusters in the group of younger adults, whereas the seed in the right insula yielded only significant results for the older adults. The left pre-SMA showed increased connectivity with subcortical structures (bilateral caudate nuclei and thalami) as well as with the left precuneus and PCC in the parietal lobe (Fig. 5A; Supplementary Table S16). For the seed in the left insula, we found a similar connectivity pattern. Significant coupling was observed with bilateral caudate nuclei and the left precuneus (Fig. 5B; Supplementary Table S17). For the older adults, the right insula showed significant coupling with the precuneus and pars orbitalis in left inferior frontal gyrus (Fig. 5C; Supplementary Table S18).

Moreover, two ROIs from the contrast Counting $>$ Semantic fluency which were associated with the DMN, the right temporal pole and the right precuneus, were used for traditional gPPI analyses. Seeding in the right temporal pole showed increased functional connectivity exclusively in the ipsilateral hemisphere. For the older adults, we found a significant cluster in inferior frontal gyrus (pars opercularis) which extended into the insula (Fig. 6A; Supplementary Table S19). For the young adults, results revealed significant clusters in the inferior frontal gyrus (pars opercularis), superior frontal gyrus, insula, and supramarginal gyrus (Fig. 6A, Supplementary Table S19). The seed in the right precuneus revealed extensive bilateral functional coupling in both age groups. For the older adults, the right precuneus showed prominent connectivity with frontal, temporal, and parietal areas in both hemispheres (Fig. 6B; Supplementary Table S20). A similar pattern emerged for the group of younger adults, albeit with a greater number of significant clusters (Fig. 6B; Supplementary Table S20). Two-sample $t$-tests did not show significant differences between groups in the PPI results for either task. 
bioRxiv preprint doi: https:/doi.org/10.1101/2020.11.06.371153; this version posted March 2, 2021. The copyright holder for this preprint (which was not certified by peer review) is the author/funder, who has granted bioRxiv a license to display the preprint in perpetuity. It is made available under aCC-BY-ND 4.0 International license.

A Seed in left pre-SMA $[-6 ; 12 ; 51]$

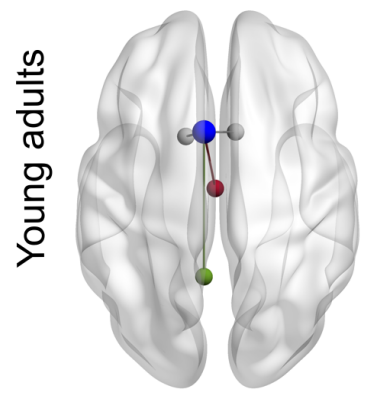

$x-14$

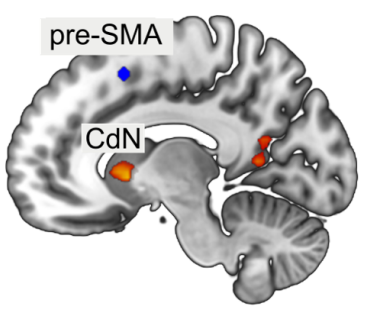

B Seed in left insula $[-31 ; 25 ; 2]$
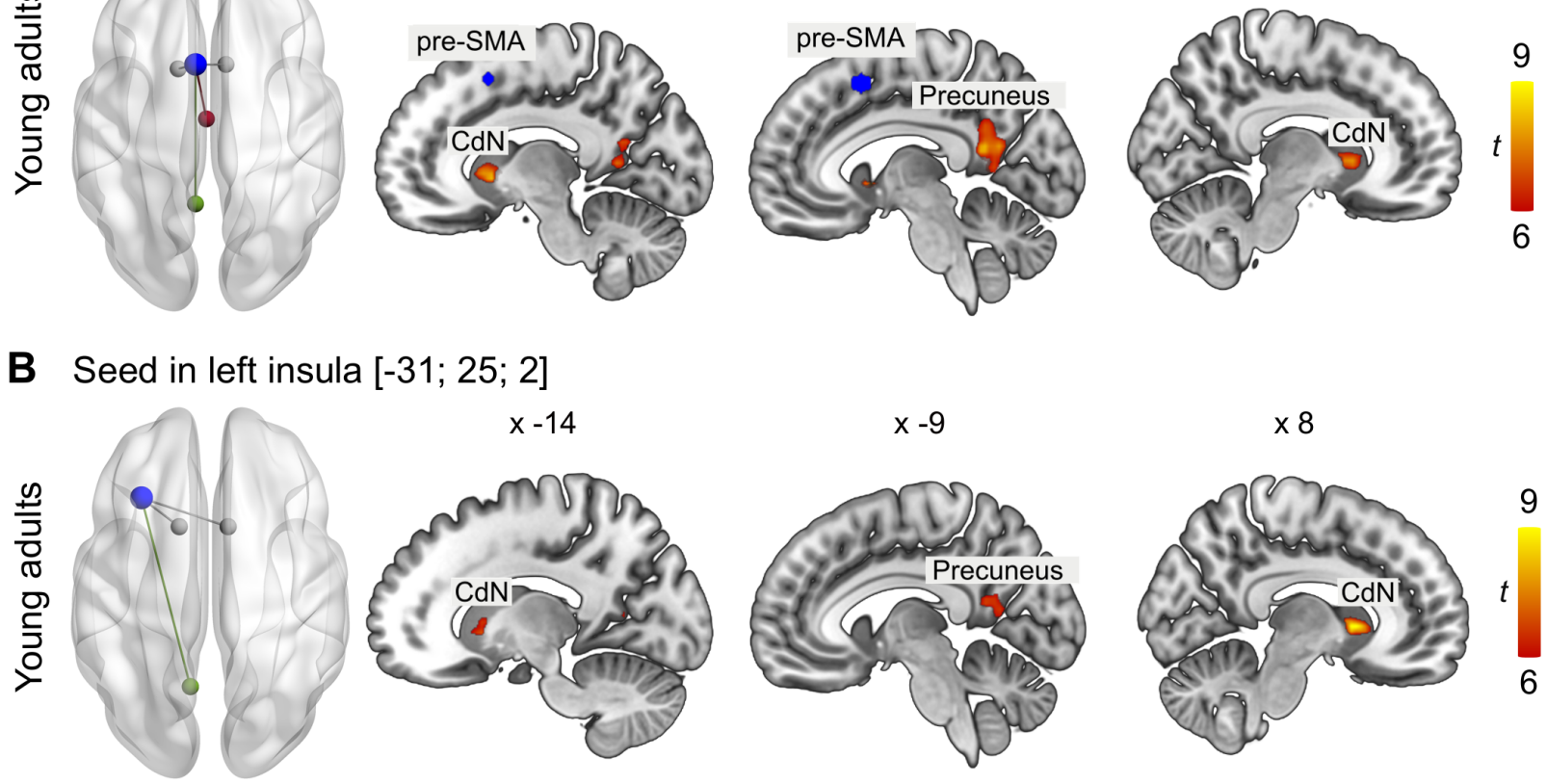

C Seed in right insula $[31 ; 27 ; 2]$

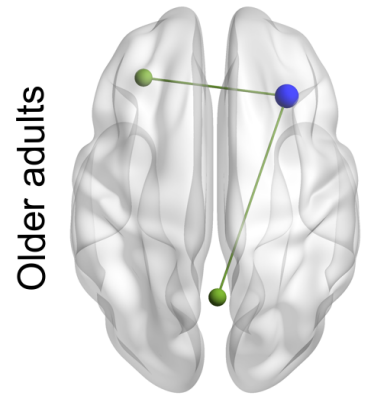

$x-31$

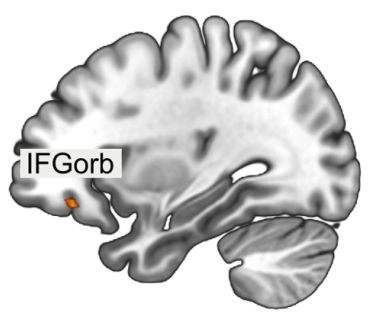

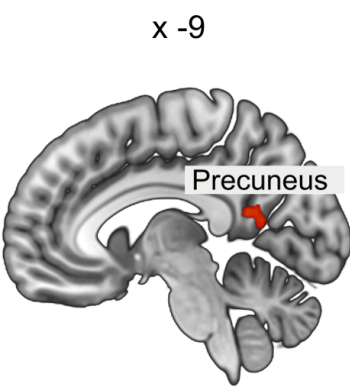

$x-6$

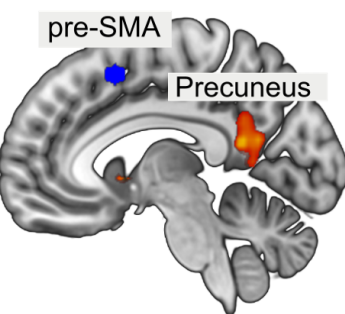

$x-9$

$x 1$

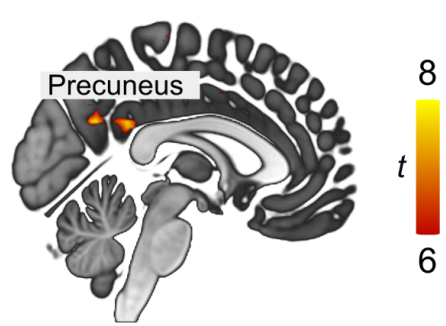

PPI Seed

MDN

DMN

Other nodes

Figure 5. Functional connectivity for seeds from contrast Semantic fluency $>$ Counting. All results are FWE-corrected at $p<0.05$ at peak level with a minimum cluster size $=20$ voxel. Abbreviations: PreSMA presupplementary motor area; $\mathrm{CdN}$ caudate nucleus; IFGorb inferior frontal gyrus, pars orbitalis. Unthresholded statistical maps are available at https://neurovault.org/collections/9072/.

Within- and between-network functional connectivity during semantic fluency

To further examine the task-related connectivity within and between the domain-general systems MDN and DMN during the semantic fluency task compared to counting, we conducted modified gPPI analyses. For each seed-to-target combination of the ROIs in MDN and DMN (Table 3), we calculated intercept-only GLMs for each age group (Fig. 7A). For older adults, the results showed significant positive functional connectivity for regions within the MDN but not for regions within the DMN. Further, the analyses revealed strong coupling 
bioRxiv preprint doi: https://doi.org/10.1101/2020.11.06.371153; this version posted March 2, 2021. The copyright holder for this preprint (which was not certified by peer review) is the author/funder, who has granted bioRxiv a license to display the preprint in perpetuity. It is made available under aCC-BY-ND 4.0 International license.

A Seed in right temporal pole $[48 ; 15 ;-31]$
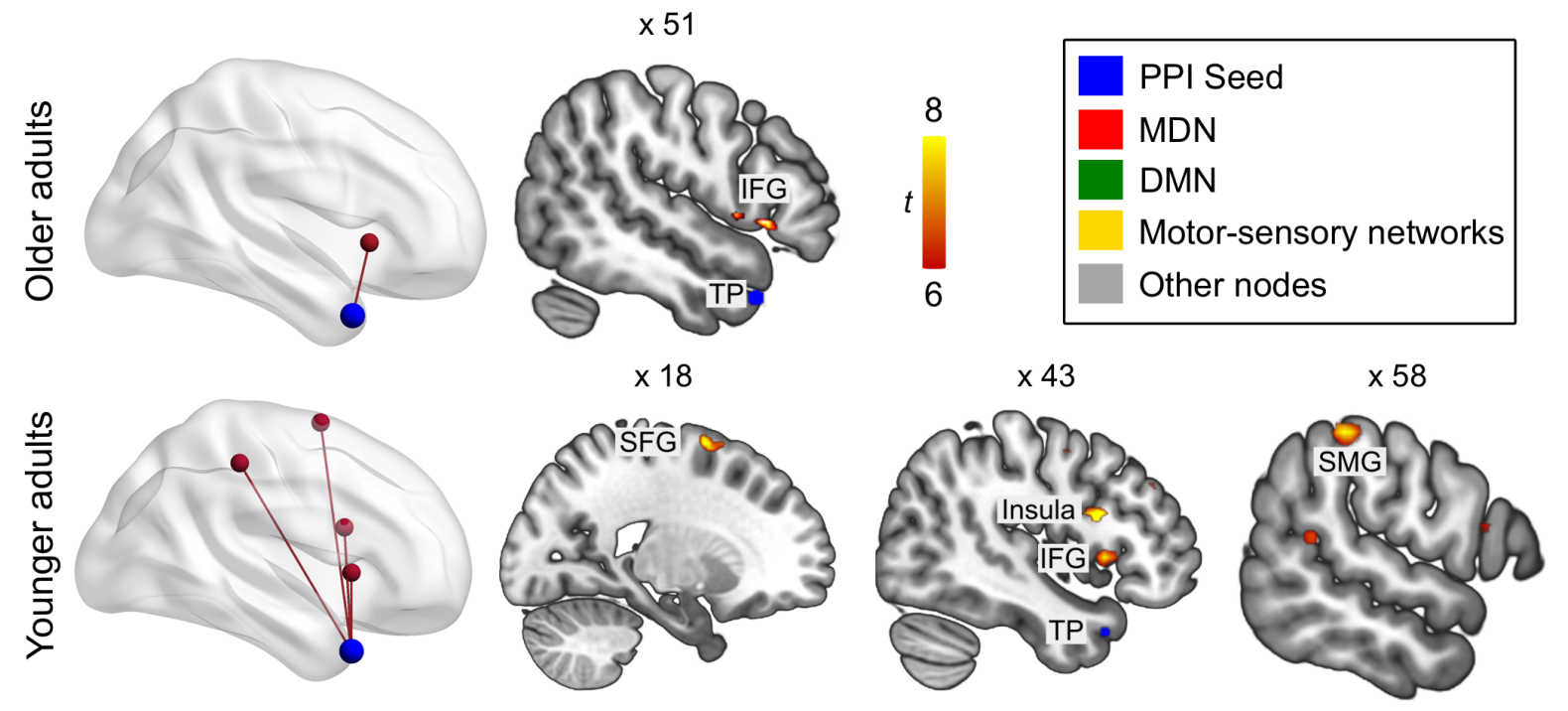

B Seed in right precuneus $[8 ;-65 ; 29]$
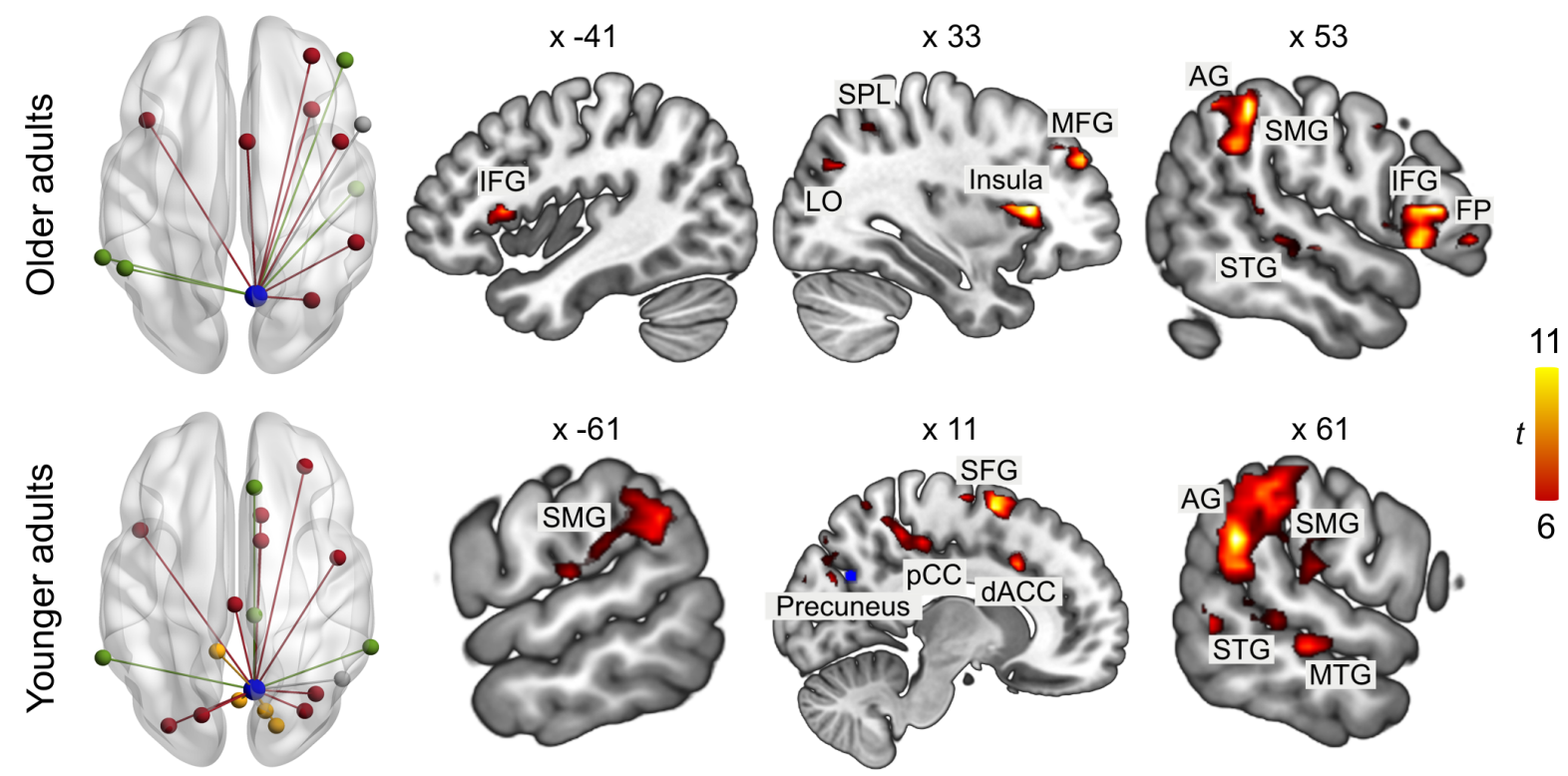

Figure 6. Functional connectivity for seeds from contrast Counting $>$ Semantic fluency. All results are FWE-corrected at $p<0.05$ at peak level with a minimum cluster size $=20$ voxel. Abbreviations: IFG inferior frontal gyrus; TP temporal pole; SFG superior frontal gyrus; SMG supramarginal gyrus; LO lateral occipital cortex; SPL superior parietal lobe; MFG middle frontal gyrus; AG angular gyrus; FP frontal pole; STG superior temporal gyrus; $\mathrm{pCC}$ posterior cingulate cortex; dACC dorsal anterior cingulate cortex; MTG middle temporal gyrus. Unthresholded statistical maps are available at https://neurovault.org/collections/9072/. 
for regions between MD and DM networks. A similar pattern was observed for young adults, albeit with overall stronger connectivity. Compared to the counting task, results showed strengthened functional connectivity within regions of the MDN and for regions between MD and DM networks during semantic fluency.

\section{Effect of age on within- and between-network functional connectivity}

We were interested whether there was an effect of age group on the within- and between network functional connectivity. To this end, each PPI network pair (within-MDN, withinDMN, and between MDN and DMN) was regressed on age (Fig. 7B; Supplementary Table S21). Multiple-comparisons correction was performed using Meff-correction. The results did not show a significant effect of age on within- and between-network functional connectivity ( $p$ 's $>0.3$ ) suggesting that the strength of functional connectivity was age-invariant.

\section{Effect of functional connectivity on in-scanner task performance}

To determine whether functional connectivity within and between regions of MDN and DMN predicted participant's in-scanner task performance, we fitted generalized mixed-effects models for accuracy and response time as outcome variables and functional connectivity, age, and their interaction terms as explanatory variables. Since the functional connectivity measures were based on our contrast of interest Semantic fluency $>$ Counting, statistical models were only fit for the behavioral results for the semantic fluency and not for the counting task. The results did not indicate significant effects of functional connectivity on accuracy (Supplementary Table S22). However, analyses revealed significant effects of functional network connectivity on response time (Fig. 7C; Supplementary Table S22 and S23). We identified main effects of within-DMN $\left(\chi^{2}=12.65, p<0.001\right)$, and betweennetwork functional connectivity $\left(\chi^{2}=29.43, p<0.001\right)$ as well as of age $\left(\chi^{2}=63.15, p<\right.$ 0.001). Beta coefficients indicated that across networks strengthened functional connectivity 
bioRxiv preprint doi: https://doi.org/10.1101/2020.11.06.371153; this version posted March 2, 2021. The copyright holder for this preprint (which was not certified by peer review) is the author/funder, who has granted bioRxiv a license to display the preprint in perpetuity. It is made available under aCC-BY-ND 4.0 International license.

A

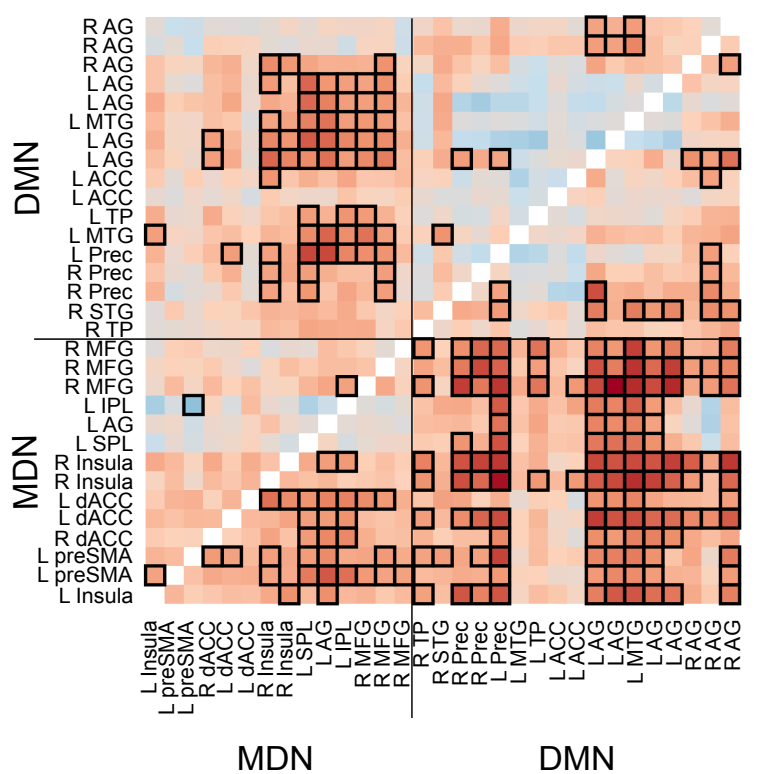

C

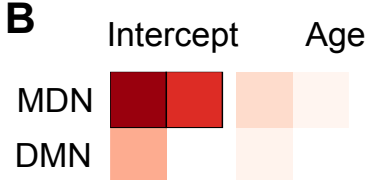

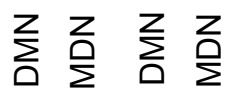

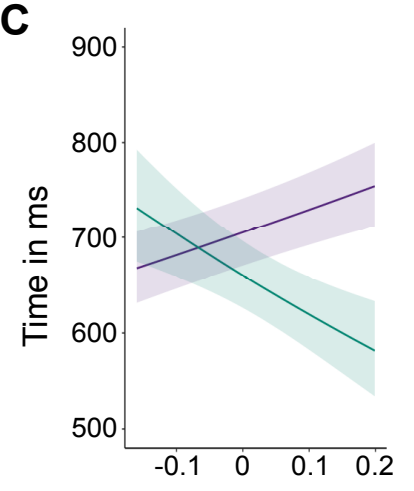

Within-MDN connectivity Within-DMN connectivity

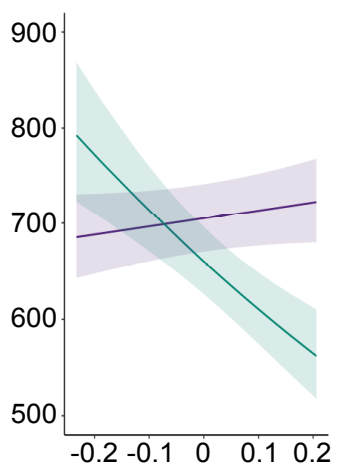

Young adults
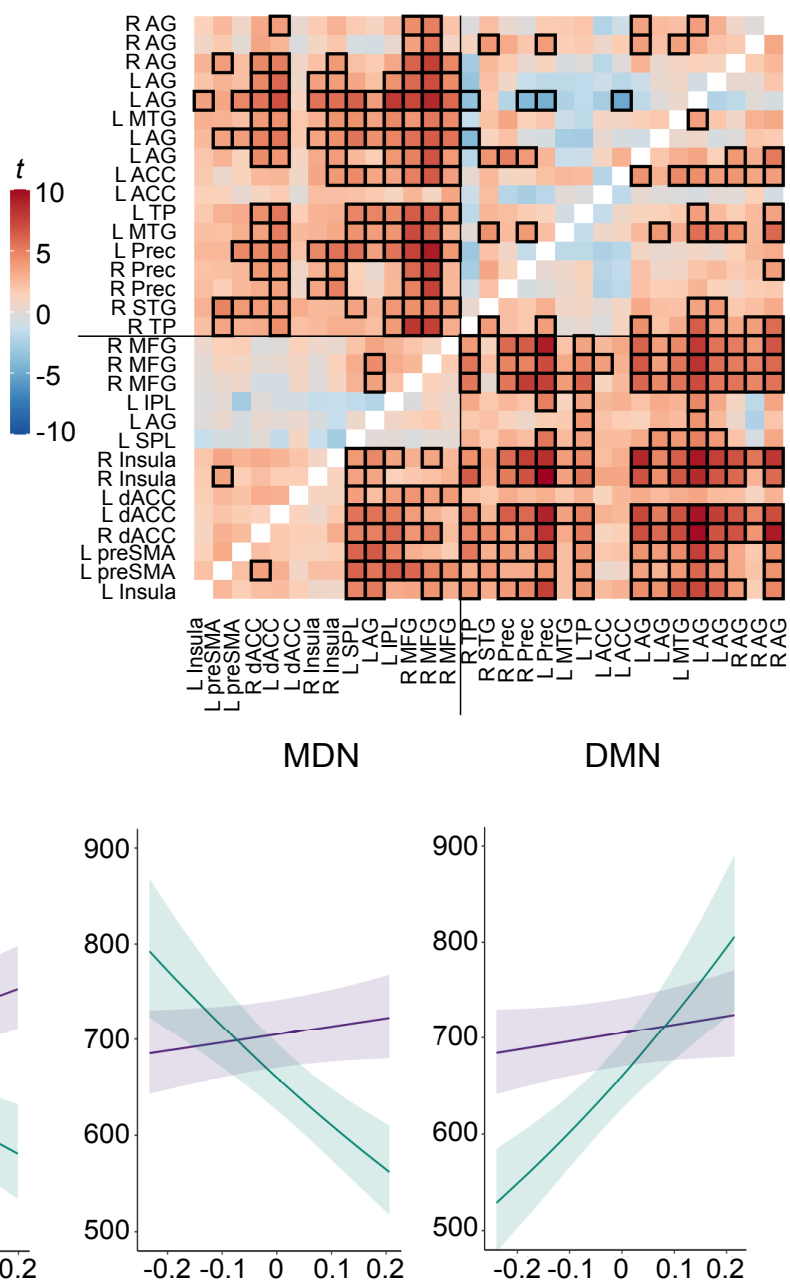

Between MDN and DMN connectivity

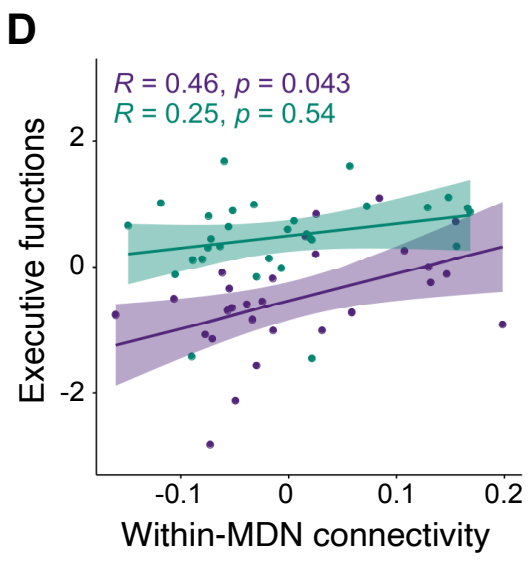

Figure 7. Functional connectivity of domain-general network regions during semantic fluency. (A) Within- and between-network functional connectivity for each seed to target combination for each age group. Heatmaps show $t$ values. Thresholded $t$ values with Meffcorrected $\alpha$ of 0.0018 are indicated with black boxes. (B) Effect of age on functional connectivity. There were no age differences in functional connectivity between or within networks. Heatmaps show $t$ values with Meff-corrected $\alpha$ of 0.02 . Significant effects are indicated with black boxes. 
(C) Significant two-way interactions between age and functional connectivity for response time during semantic fluency. (D) Correlation analyses between functional connectivity measures and neuropsychological factors. For within-MDN connectivity, only older adults showed a significant correlation with executive functions, while only young adults showed a significant correlation between semantic memory and between-network functional connectivity. Abbreviations: R right hemisphere; L left hemisphere; AG angular gyrus; MTG middle temporal gyrus; ACC anterior cingulate cortex; TP temporal pole; Prec Precuneus; STG superior temporal gyrus; MFG middle frontal gyrus; IPL inferior parietal lobe; SPL superior parietal lobe; dACC dorsal anterior cingulate cortex; preSMA presupplementary motor area; MDN Multiple-demand network; DMN Default mode network.

was associated with slower response times and that young adults performed generally faster than older adults which confirmed our behavioral results. Moreover, significant interactions between age and within-MDN $\left(\chi^{2}=32.84, p<0.001\right)$, within-DMN $\left(\chi^{2}=37.33, p<0.001\right)$, and between-network functional connectivity $\left(\chi^{2}=24.78, p<0.001\right)$ were found. Post-hoc tests showed that age group had a different effect on within- and between-network functional connectivity. While response time increased with strengthened connectivity in the MDN and the DMN for older adults, the opposite pattern was observed for young adults who responded faster when within-network functional connectivity increased (all $p<0.001$ ). For functional connectivity between MDN and DMN, stronger coupling predicted slower responses in both age groups, albeit with young adults showing a significantly steeper positive slope than older adults $(p<0.001$; Fig. 7C).

\section{Effect of functional connectivity on cognitive performance and semantic memory}

We were interested in the relationship between functional connectivity and general cognitive and semantic memory performance which were assessed via neuropsychological tests outside of the scanner. Since some tests showed high collinearity, we first performed a factor analysis on the data of both age groups together. Results identified two factors: A cognitive performance factor with high loadings on trail making tests $\mathrm{A}(0.8)$ and $\mathrm{B}(0.71)$, the digit symbol substitution test (0.73), and the reading span test (0.45), and a semantic memory factor with high loadings on the spot-the-word test (0.5) and the two verbal fluency tests for 
hobbies (0.44) and surnames (0.98). Individual factor scores for participants were extracted and subsequently correlated with functional connectivity measures. The resulting $p$-values were corrected for multiple comparisons using Bonferroni-correction $(p=0.05 / 3$ functional connectivity parameters $=0.017)$. First, we used partial Pearson correlations to test for a relation between connectivity and cognitive performance while controlling for the effect of age. Results revealed a significant positive correlation between executive functions and within-MDN functional connectivity $(r=0.36, p=0.018)$. Second, we calculated Pearson correlations within each age group. For older adults, we found a significant positive correlation between cognitive performance and within-MDN functional connectivity $(r=$ $0.46, p=0.043 ;$ Fig. 7D). For young adults, results showed a significant positive correlation between semantic memory and functional connectivity between MDN and DMN $(r=0.48, p$ $=0.024$; Fig. 7D).

Taken together, functional connectivity within- and between-MD and DM network regions was associated with efficiency during the experimental task as well as general cognitive and semantic performance in both age groups. The effect of functional connectivity on response time was moderated by age with young adults profiting from a strengthened within-network connectivity whereas older adults showed a decline in response speed. Furthermore, both age groups performed slower when functional connectivity between both domain-general systems increased. Finally, functional connectivity was differently related to out-of-scanner tasks in both age groups. While analyses revealed a positive association between cognitive performance and within-MDN functional connectivity in older adults, between-network functional connectivity showed a positive effect on semantic memory in young adults. 


\section{Discussion}

The current study set out to describe the effects of aging on the interplay of domain-specific and domain-general neural networks in semantic cognition. By contrasting a semantic fluency task with a low-level verbal control task in an fMRI experiment, we delineated two distinct task-related networks which displayed strong overlap with the domain-general MD and DM systems. Using task-based connectivity analyses, our findings point towards a strong interaction of these networks during verbal semantic processing across age groups and lend support to the notion that integration between usually anti-correlated functional networks increases for tasks that require cognitive control (Shine et al., 2016). Importantly, our results provide new insights into the impact of age on the functional coupling within and between MDN and DMN regions when semantic knowledge is retrieved in a goal-directed manner from memory. In line with a recent suggestion that additional recruitment of the prefrontal cortex in older adulthood might not reflect compensation but rather reduced efficiency or specificity (Morcom and Henson, 2018), we show here that increased in-phase synchronization of task-relevant networks is generally associated with a decline in task efficiency in older adults whereas young adults capitalize more on strengthened functional connectivity. This finding sheds new light on the frequently reported pattern of strengthened between-network functional connectivity in older adults at rest (Chan et al., 2014; Geerligs et al., 2015; Spreng et al., 2016).

Our task paradigm revealed two distinct functional networks for semantic fluency and counting. The main effect of semantic fluency displayed a predominantly left lateralized fronto-temporo-parietal network for both age groups with additional activation peaks in right frontal and temporal areas, bilateral caudate nuclei, and the cerebellum. These results align well with previous investigations that applied a semantic fluency paradigm (Baciu et al., 2016; Birn et al., 2010; Marsolais et al., 2014; Meinzer et al., 2009, 2012a, 2012b; Nagels et 
al., 2012; Vitali et al., 2005; Wagner et al., 2014; Whitney et al., 2009). The main effect of the counting task was evident in both groups in bilateral activation of sensorimotor cortices and the cerebellum which is consistent with previous studies that used an automated speech task (e.g., Birn et al., 2010; Geranmayeh et al., 2014; Marsolais et al., 2014). Further, older adults showed recruitment of the pre-supplementary motor area which could reflect increased cognitive demands for this age group while keeping track of the numbers during counting. In the direct comparison of both tasks, semantic fluency elicited a network that resembled the main effect of the task minus activity in pre- and postcentral gyri in both age groups which corroborates the functional role of this network in spoken language beyond low-level sensorimotor aspects (Geranmayeh et al., 2014). Significant activation for the counting task compared to semantic fluency was evident in a mainly right lateralized network. Previous studies have suggested that neural networks for highly overlearned automated speech tasks are either right-lateralized in healthy participants (Sidtis et al., 2009; Vanlancker-Sidtis et al., 2003) or show less left lateralization than semantically rich language production tasks (Bookheimer et al., 2000; Petrovich Brennan et al., 2007). Further evidence stems from the common observation that automated speech (e.g., counting) is often preserved in patients who suffer from aphasia after a left hemisphere stroke (Vanlancker-Sidtis et al., 2003).

Despite the semantic nature of the task, we found that the strongest activation clusters for semantic fluency were located in the domain-general MD system in both age groups. These results are in line with previous studies that applied a similar task (e.g., Basho et al., 2007; Lurito et al., 2000) and highlight the strong executive aspect of this paradigm. There is emerging evidence on the overlap of language-specific regions like the left inferior frontal gyrus (IFG) with networks that are implicated in domain-general executive processing (Fedorenko et al., 2012) and semantic control processes (Jackson et al., 2021; Noonan et al., 2013; Thompson-Schill et al., 1997). A recent meta-analysis demonstrated an overlap of 
some regions of the semantic control network with the MDN thus emphasizing the role of domain-general control in language processing (Jackson, 2021). Here, we observed that semantic fluency predominantly activated the domain-general regions of the semantic control network, like the pre-SMA and the dorsomedial prefrontal cortex including dorsal IFG, and only a small part of domain-specific semantic control (ventral IFG). Hence, the domaingeneral control regions may not be language-specific but appear to strongly contribute to a task that requires goal-directed controlled access to semantic memory while monitoring the verbal articulation of words that match the semantic categories. The scope of the present investigation was confined to the age-dependent contribution of domain-general systems to semantic cognition. Nonetheless, their interaction with the semantic network remains certainly an important question for future research. Further support for the contribution of executive functions to semantic fluency stems from behavioral studies that associated cognitive flexibility, inhibition, working memory, and attention with successful performance (Aita et al., 2018; Amunts et al., 2020; Gordon et al., 2018). For both age groups, peak clusters of counting were found in the posterior DMN which is in line with our expectation of a low-level language production task in comparison to the more demanding semantic fluency task.

Our whole-brain functional connectivity results based on traditional gPPI analyses showed that regions in the domain-general MD and DM systems strongly interact during a semantic word retrieval task compared to counting across both age groups. This was true for seeds coming from the MDN as well as the DMN. This finding is in line with previous studies that reported task-specific functional coupling of cognitive control regions with the $\mathrm{DMN}$, most notably the posterior cingulate cortex (PCC)/precuneus, especially in tasks requiring controlled access to semantic memory (Krieger-Redwood et al., 2016; Smith et al., 2016). Remarkably, the PCC/precuneus was the only region in our study that showed 
functional connectivity with all seeds from the MDN and displayed extensive functional coupling with multiple nodes in the DMN as well as other neural networks in both age groups. This finding stresses its role as a cortical hub connecting networks to support complex behavior (Leech et al., 2012). The functional coupling of MDN and DMN is especially interesting in light of our functional results where we observed significant deactivation in regions of the DMN during semantic fluency in both age groups. It corroborates the notion that networks that are anti-correlated during task can still show functional integration in contextually relevant situations to facilitate goal-directed behavior (Krieger-Redwood et al., 2016; Spreng et al., 2014).

We gained further insight into the task-related functional integration of MD and DM network regions by our analyses of phase synchronization within and between both domaingeneral systems. Our results show that functional coupling within the MDN and between the MDN and the DMN strengthened with an increase of task load, which was true independent of age. First, the positive coupling within regions of the MDN is in line with our univariate results for semantic fluency: here, we found that the strongest activation clusters were located in the MDN in both age groups, thus confirming the necessary engagement of this network for successful task performance. Second, the strong in-phase synchronicity between regions of the MD and DM network for semantic fluency compared to the control task complement our PPI results which showed a strong interaction of both domain-general systems. This is line with the notion that the integration of the DMN is relevant for successful task processing in memory-guided cognition (Smith et al., 2016; Vatansever et al., 2015), especially when access to semantic memory is required (Wirth et al., 2011; Krieger-Redwood et al., 2019).

Interestingly, our results on whole-brain as well as within- and between-network functional connectivity did not reveal an effect of age. There is an extant literature describing age-related changes in connectivity in resting state networks with the most common 
observation of decreased within- and increased between-network functional connectivity (Chan et al., 2014; Ferreira et al., 2016; Geerligs et al., 2015; Grady et al., 2016; Ng et al., 2016; Zonneveld et al., 2019). However, results are more inconsistent for task-related changes in functional connectivity with age. Across a range of cognitive tasks, studies reported a similar pattern as for resting state investigations (Geerligs et al., 2014; Spreng et al., 2016), no changes for within- but only for between-network connectivity (Gallen et al., 2016; Grady et al., 2016), as well as age invariance (Pongpipat et al., 2020; Trelle et al., 2019). In the domain of semantic cognition, findings are sparse with one study observing reduced within-network integration for a semantic fluency task which was not associated with a poorer performance in older adults (Marsolais et al., 2014). There are two possible explanations for the present age invariance in functional connectivity. First, the group of older adults in our study might have been too young to detect changes in functional connectivity. Longitudinal studies on cognitive aging showed that a turning point in functional coupling takes place around the age of 65-70 years ( $\mathrm{Ng}$ et al., 2016; Zonneveld et al., 2019). Thus, although the young adults in our study displayed numerically greater and stronger functional coupling than the older adults, the overall pattern was too similar in both groups. Second, the lack of an age effect on functional connectivity might be related to the semantic nature of our fluency paradigm. Semantic tasks have been shown to require functional coupling between cognitive control as well as DM regions like the PCC/precuneus which has been implicated in semantic cognition even in young adults (Krieger-Redwood et al., 2016). Hence, this might have aggravated the possibility of observing the frequently reported increase of between-network functional connectivity in older adults and underlines the necessity for more task-based investigations in the future to better understand the picture of neurocognitive aging. 
Intriguingly, despite the observed age invariance, functional connectivity had different effects on in-scanner task performance and cognitive functioning in both age groups. Our results show that older adults did not capitalize on strengthened functional connectivity in the same way as young adults. This was the case for functional connectivity within the MDN and the DMN where an increase of connectivity was associated with slower performance in the semantic fluency task in older adults but with faster performance in young adults (Fig. 8A). In contrast, strengthened between-network functional connectivity led to a slower performance in both age groups, with a stronger effect for young adults (Fig. 8B). Considering our whole-brain connectivity results that showed strong positive coupling between both networks during semantic fluency, this decrease in efficiency might reflect the more effortful communication between task-relevant networks compared to within-network coupling, hence leading to a slower performance across age groups. Interestingly, despite the negative effect on task efficiency, increased functional coupling between MD and DM had a positive effect on semantic memory in young but not in older adults. For the latter group, strengthened connectivity within MD regions was associated with better cognitive performance, albeit still at a significantly lower level of performance than in young adults.

Overall, our findings on the age-dependent relevance of functional connectivity to behavior are in line with theories of neurocognitive aging that suggest a reduced efficiency of neural networks with age (Davis et al., 2012; Geerligs et al., 2014; Shafto and Tyler, 2014). Although older adults rely on similar neural networks as young adults for task processing, they cannot equally capitalize on them. Young adults increased their performance as well as their processing efficiency with strengthened in-phase synchronization of task-relevant networks while older adults showed improvements in cognitive performance but not in efficiency with increased connectivity. Our results thus provide new insights into the behavioral relevance of the frequently observed pattern of neural dedifferentiation (Baltes 


\section{A Within-network functional connectivity}

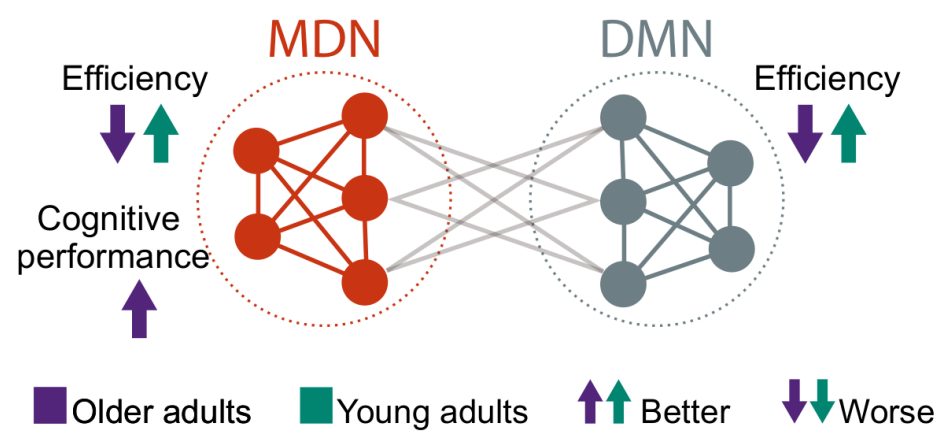

B Between-network functional connectivity

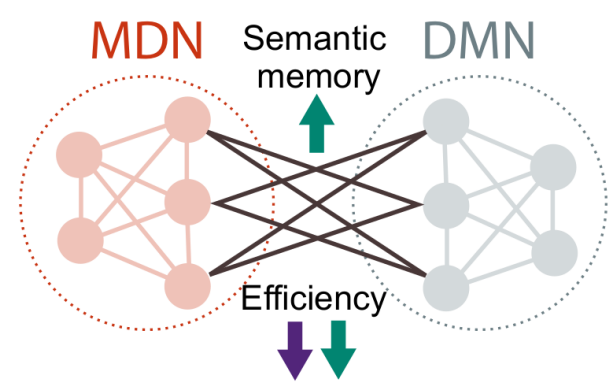

Figure 8. The different effects of within- and between network functional connectivity on task performance in each age group. (A) Young adults improved their efficiency in the form of faster response times whereas older adults performed slower when functional connectivity within the MDN or DMN increased. Moreover, strengthened connectivity within the MDN was related to a better performance in executive measures for older adults. (B) Strengthened between-network functional connectivity led to a decline in efficiency in both age groups. However, it was also associated with an improved performance in semantic memory only for young adults. Abbrev.: MDN Multiple-demand network; DMN Default mode network.

and Lindenberger, 1997; Grady, 2012; Park et al., 2004) showing that older adults do not engage task-relevant networks in the same beneficial way as young adults. This is especially relevant in the context of semantic cognition where, according to the DECHA framework, increased semantic knowledge with age could lead to a performance advantage (Spreng and Turner, 2019). Here, we show that this is not the case in a task that requires an efficient use of control systems while accessing semantic memory; thus, lending support to the notion that older adults are less flexible in the goal-directed functional coupling of executive and default resources (Spreng and Turner, 2019).

Our findings on age-related differences in cortical activation for both tasks further underline the observed reduced efficiency of neural networks in older adults. The comparison of age groups for the semantic fluency task compared to rest revealed stronger activation only for the older adults. Significant clusters comprised hubs of the MDN as well as the right inferior frontal gyrus which has been emphasized in studies on semantic fluency in aging before (Meinzer et al., 2009; Meinzer et al., 2012a; Meinzer et al., 2012b; Nagels et al., 2012). Remarkably, the interaction between both tasks and age revealed significant effects 
only for the young adults who displayed stronger activation of frontal key regions of the MDN including the pre-SMA and dACC for semantic fluency. This finding suggests a pattern of increased processing efficiency which was reflected by faster response times compared to the older group. It converges with previous studies on language production and comprehension that associated greater activation in the prefrontal cortex with an increased task demand in young adults (Fu et al., 2002; Thompson-Schill et al., 1997; Whitney et al., 2009). The supplementary activation of MDN regions in older adults for the semantic fluency task compared to rest aligns with a meta-analysis on semantic cognition that found greater activity in areas of the MDN with older age (Hoffman and Morcom, 2018). The nature of this upregulation in brain activity in older adults has been subject of some debate (Cabeza et al., 2018; Morcom and Johnson, 2015). Here, we observed additional activation in the older adults while they performed poorer than the young adults during the more demanding semantic fluency task. In light of the additional beneficial activation of frontal MDN regions in the young adults, the observed upregulation in the older adults seems to further support the idea of age-related reduced efficiency of neural responses (Nyberg et al., 2014) leading to a stronger involvement of executive control at a lower level of task demand (Gallen et al, 2016; Hakun et al., 2015). This interpretation is backed up by the observed age-related differences in task-dependent activation of the MD and DM regions. During semantic fluency, older adults showed less deactivation of the DMN than young adults while during counting, the MDN was less deactivated in older than in young adults. Thus, in line with our functional connectivity results, older adults recruit similar neural resources as young adults, albeit at a lower level of processing efficiency which lends additional support to the hypothesis of dedifferentiation (Morcom and Henson, 2018; Park et al., 2004).

It should be noted that our results did not show a consistent effect of the intended modulation of task difficulty within the semantic fluency task on neural activation patterns. 
This could be related to the limited number of items participants had to produce for each category. A recent behavioral investigation on semantic fluency showed that the amount of correct responses continuously decreases with time (Gordon et al., 2018). Thus, although the effect of difficulty was present in the behavioral results in the form of reduced accuracy and slower responses, we assume that 9 trials per category were not enough to establish this effect on the neural level.

\section{Conclusion}

In conclusion, the current study sheds light on the age-dependent contribution of the domaingeneral MD and DM systems during a verbal semantic fluency task. While univariate results revealed strong activity in the MDN during task processing, functional connectivity analyses demonstrated a strong interaction between the MDN and the DMN for semantic fluency. This finding corroborates the notion that usually anti-correlated networks integrate for successful task processing, especially when access to semantic memory is required. Although the strength of functional connectivity within- and between-networks was age-invariant, it had a different behavioral relevance in both age groups. Only the young adults engaged taskrelevant networks in a beneficial way. This was evident in the form of better processing efficiency during semantic fluency and generally improved semantic memory. In older adults, strengthened functional connectivity within the MDN had a positive effect on cognitive performance, albeit older adults still performed at a lower level than young adults. Our results provide new insights into the concept of age-related reduced efficiency in the domain of semantic cognition and inform about the behavioral relevance of the frequently observed pattern of neural dedifferentiation. 


\section{Acknowledgments}

The authors would like to thank the medical technical assistants of MPI CBS for their support with data acquisition, and Annika Dunau, Caroline Duchow, and Rebekka Luckner for their support with transcriptions of recordings.

\section{Funding}

SM held a stipend by the German Academic Scholarship Foundation (Studienstiftung des deutschen Volkes). DS was supported by the Deutsche Forschungsgemeinschaft (SA 1723/51) and the James S. McDonnell Foundation (Understanding Human Cognition, \#220020292). GH was supported by the Lise Meitner excellence program of the Max Planck Society.

\section{Code and Data Availability}

All behavioral data as well as extracted beta weights generated or analyzed during this study have been deposited in a public repository on Gitlab https://gitlab.gwdg.de/functionalconnectivityaging/mdn_lang. This repository also holds all self-written analysis code used for this project. Unthresholded statistical group maps for fMRI and gPPI results are made publicly available on NeuroVault:

https://neurovault.org/collections/9072/. Raw and single subject neuroimaging data are protected under the General Data Protection Regulation (EU) and can only be made available from the authors upon reasonable request.

\section{Competing Interests}

The authors declare that no competing interests exist. 


\section{References}

Aita SL, Beach JD, Taylor SE, Borgogna NC, Harrell MN, Hill BD. 2018. Executive, language, or both? An examination of the construct validity of verbal fluency measures. Applied Neuropsychology: Adult.:1-11. doi:10.1080/23279095.2018.1439830.

Amunts J, Camilleri JA, Eickhoff SB, Heim S, Weis S. 2020. Executive functions predict verbal fluency scores in healthy participants. Sci Rep. 10(1):11141. doi:10.1038/s41598020-65525-9.

Andrews-Hanna JR, Snyder AZ, Vincent JL, Lustig C, Head D, Raichle ME, Buckner RL. 2007. Disruption of Large-Scale Brain Systems in Advanced Aging. Neuron. 56(5):924935. doi:10.1016/j.neuron.2007.10.038.

Aschenbrenner S, Tucha O, Lange KW. 2000. Regensburger Wortflüssigkeits-Test. Göttingen: Hogrefe.

Baciu M, Boudiaf N, Cousin E, Perrone-Bertolotti M, Pichat C, Fournet N, Chainay H, Lamalle L, Krainik A. 2016. Functional MRI evidence for the decline of word retrieval and generation during normal aging. AGE. 38(1):3. doi:10.1007/s11357-015-9857-y.

Baddeley A, Emslie H, Nimmo-Smith I. 1993. The Spot-the-Word test: a robust estimate of verbal intelligence based on lexical decision. Br J Clin Psychol. 32(1):55-65. doi:10.1111/j.2044-8260.1993.tb01027.x.

Balota DA, Dolan PO, Duchek JM. 2000. Memory changes in healthy older adults. In: The Oxford handbook of memory. New York, NY, US: Oxford University Press. p. 395-409.

Baltes PB, Lindenberger U. 1997. Emergence of a powerful connection between sensory and cognitive functions across the adult life span: a new window to the study of cognitive aging? Psychol Aging. 12(1):12-21. doi:10.1037//0882-7974.12.1.12. 
Basho S, Palmer ED, Rubio MA, Wulfeck B, Müller R-A. 2007. Effects of generation mode in fMRI adaptations of semantic fluency: Paced production and overt speech.

Neuropsychologia. 45(8):1697-1706. doi:10.1016/j.neuropsychologia.2007.01.007.

Bates D, Mächler M, Bolker B, Walker S. 2015. Fitting linear mixed-effects models using lme4. Journal of Statistical Software. 67(1):1-48. doi:10.18637/jss.v067.i01.

Beck AT, Steer RA, Ball R, Ranieri W. 1996. Comparison of Beck Depression Inventories IA and -II in psychiatric outpatients. J Pers Assess. 67(3):588-597. doi: $10.1207 / \mathrm{s} 15327752 \mathrm{jpa6703} \mathrm{13.}$

Birn RM, Kenworthy L, Case L, Caravella R, Jones TB, Bandettini PA, Martin A. 2010.

Neural systems supporting lexical search guided by letter and semantic category cues: A self-paced overt response fMRI study of verbal fluency. NeuroImage. 49(1):1099-1107. doi:10.1016/j.neuroimage.2009.07.036.

Bookheimer SY, Zeffiro TA, Blaxton TA, Gaillard W, Theodore WH. 2000. Activation of language cortex with automatic speech tasks. Neurology. 55(8):1151-1157. doi:10.1212/WNL.55.8.1151.

Brett M, Anton J-L, Valabregue R, Poline J-B. 2002. Region of Interest Analysis Using an SPM Toolbox. Neuroimage. 16. doi:10.1016/S1053-8119(02)90013-3.

Cabeza R. 2002. Hemispheric asymmetry reduction in older adults: The HAROLD model. Psychology and Aging. 17(1):85-100. doi:10.1037/0882-7974.17.1.85.

Cabeza R, Albert M, Belleville S, Craik FIM, Duarte A, Grady CL, Lindenberger U, Nyberg L, Park DC, Reuter-Lorenz PA, et al. 2018. Maintenance, reserve and compensation: the cognitive neuroscience of healthy ageing. Nature Reviews Neuroscience. 19(11):701-710. doi:10.1038/s41583-018-0068-2. 
Chan MY, Park DC, Savalia NK, Petersen SE, Wig GS. 2014. Decreased segregation of brain systems across the healthy adult lifespan. PNAS. 111(46):E4997-E5006. doi:10.1073/pnas.1415122111.

Cohen-Shikora ER, Balota DA. 2016. Visual word recognition across the adult lifespan. Psychology and Aging. 31(5):488-502. doi:10.1037/pag0000100.

Dale AM, Fischl B, Sereno MI. 1999. Cortical Surface-Based Analysis: I. Segmentation and Surface Reconstruction. NeuroImage. 9(2):179-194. doi:10.1006/nimg.1998.0395.

Damoiseaux JS. 2017. Effects of aging on functional and structural brain connectivity. NeuroImage. 160:32-40. doi:10.1016/j.neuroimage.2017.01.077.

Damoiseaux JS, Beckmann CF, Arigita EJS, Barkhof F, Scheltens P, Stam CJ, Smith SM, Rombouts S a. RB. 2008. Reduced resting-state brain activity in the "default network" in normal aging. Cereb Cortex. 18(8):1856-1864. doi:10.1093/cercor/bhm207.

Daneman M, Carpenter PA. 1980. Individual differences in working memory and reading. Journal of Verbal Learning and Verbal Behavior. 19(4):450-466. doi:10.1016/S0022$\underline{5371(80) 90312-6 .}$

Derringer J. 2018. A simple correction for non-independent tests. PsyArXiv. [accessed 2020 Aug 24]. https://osf.io/f2tyw.

Eickhoff SB, Stephan KE, Mohlberg H, Grefkes C, Fink GR, Amunts K, Zilles K. 2005. A new SPM toolbox for combining probabilistic cytoarchitectonic maps and functional imaging data. Neuroimage. 25(4):1325-1335. doi:10.1016/j.neuroimage.2004.12.034.

Esteban O, Markiewicz CJ, Blair RW, Moodie CA, Isik AI, Erramuzpe A, Kent JD, Goncalves M, DuPre E, Snyder M, et al. 2019. fMRIPrep: a robust preprocessing pipeline for functional MRI. Nat Methods. 16(1):111-116. doi:10.1038/s41592-018-0235-4. 
Fedorenko E, Duncan J, Kanwisher N. 2012. Language-Selective and Domain-General Regions Lie Side by Side within Broca’s Area. Current Biology. 22(21):2059-2062. doi:10.1016/j.cub.2012.09.011.

Fedorenko E, Duncan J, Kanwisher N. 2013. Broad domain generality in focal regions of frontal and parietal cortex. Proceedings of the National Academy of Sciences. 110(41):16616-16621. doi:10.1073/pnas.1315235110.

Feinberg DA, Moeller S, Smith SM, Auerbach E, Ramanna S, Glasser MF, Miller KL, Ugurbil K, Yacoub E. 2010. Multiplexed Echo Planar Imaging for Sub-Second Whole Brain FMRI and Fast Diffusion Imaging. PLOS ONE. 5(12):e15710. doi:10.1371/journal.pone.0015710.

Ferreira LK, Regina ACB, Kovacevic N, Martin M da GM, Santos PP, Carneiro C de G, Kerr DS, Amaro E, McIntosh AR, Busatto GF. 2016. Aging Effects on Whole-Brain Functional Connectivity in Adults Free of Cognitive and Psychiatric Disorders. Cereb Cortex. 26(9):3851-3865. doi:10.1093/cercor/bhv190.

Folstein MF, Folstein SE, McHugh PR. 1975. "Mini-mental state": A practical method for grading the cognitive state of patients for the clinician. Journal of Psychiatric Research. 12(3):189-198. doi:10.1016/0022-3956(75)90026-6.

Fonov V, Evans A, McKinstry R, Almli C, Collins D. 2009. Unbiased nonlinear average ageappropriate brain templates from birth to adulthood. NeuroImage. 47:S102. doi:10.1016/S1053-8119(09)70884-5.

Fu CHY, Morgan K, Suckling J, Williams SCR, Andrew C, Vythelingum GN, McGuire PK. 2002. A Functional Magnetic Resonance Imaging Study of Overt Letter Verbal Fluency Using a Clustered Acquisition Sequence: Greater Anterior Cingulate Activation with Increased Task Demand. NeuroImage. 17(2):871-879. doi:10.1006/nimg.2002.1189. 
Gallen CL, Turner GR, Adnan A, D’Esposito M. 2016. Reconfiguration of brain network architecture to support executive control in aging. Neurobiology of Aging. 44:42-52. doi:10.1016/j.neurobiolaging.2016.04.003.

Geerligs L, Renken RJ, Saliasi E, Maurits NM, Lorist MM. 2015. A Brain-Wide Study of Age-Related Changes in Functional Connectivity. Cereb Cortex. 25(7):1987-1999. doi:10.1093/cercor/bhu012.

Geranmayeh F, Wise RJS, Mehta A, Leech R. 2014. Overlapping Networks Engaged during Spoken Language Production and Its Cognitive Control. Journal of Neuroscience. 34(26):8728-8740. doi:10.1523/JNEUROSCI.0428-14.2014.

Glauer M, Häusig S, Krüger M, Betsch T, Renkewitz F, Sedlmeier P, Winkler I. 2007. Typizitätsnormen für Vertreter von 30 Kategorien. Neurolinguistik.:12.

Gordon JK, Young M, Garcia C. 2018. Why do older adults have difficulty with semantic fluency? Aging, Neuropsychology, and Cognition. 25(6):803-828. doi:10.1080/13825585.2017.1374328.

Gorgolewski KJ, Esteban O, Ellis DG, Notter MP, Ziegler E, Johnson H, Hamalainen C, Yvernault B, Burns C, Manhães-Savio A, et al. 2017. Nipype: A Flexible, Lightweight And Extensible Neuroimaging Data Processing Framework In Python. 0.13.1. Zenodo. [accessed 2020 Oct 19]. https://zenodo.org/record/581704.

Grady C. 2012. The cognitive neuroscience of ageing. Nat Rev Neurosci. 13(7):491-505. doi: $\underline{10.1038 / \mathrm{nrn} 3256 .}$.

Grady C, Sarraf S, Saverino C, Campbell K. 2016. Age differences in the functional interactions among the default, frontoparietal control, and dorsal attention networks. Neurobiology of Aging. 41:159-172. doi:10.1016/j.neurobiolaging.2016.02.020. 
Hakun JG, Zhu Z, Brown CA, Johnson NF, Gold BT. 2015. Longitudinal alterations to brain function, structure, and cognitive performance in healthy older adults: A fMRI-DTI study. Neuropsychologia. 71:225-235. doi:10.1016/j.neuropsychologia.2015.04.008.

Halai AD, Welbourne SR, Embleton K, Parkes LM. 2014. A comparison of dual gradientecho and spin-echo fMRI of the inferior temporal lobe. Hum Brain Mapp. 35(8):41184128. doi:10.1002/hbm.22463.

Hedden T, Gabrieli JDE. 2004. Insights into the ageing mind: a view from cognitive neuroscience. Nat Rev Neurosci. 5(2):87-96. doi: $\underline{10.1038 / \text { nrn1323. }}$.

Heinzel S, Metzger FG, Ehlis A-C, Korell R, Alboji A, Haeussinger FB, Hagen K, Maetzler W, Eschweiler GW, Berg D, et al. 2013. Aging-related cortical reorganization of verbal fluency processing: a functional near-infrared spectroscopy study. Neurobiology of Aging. 34(2):439-450. doi:10.1016/j.neurobiolaging.2012.05.021.

Hoffman P, Morcom AM. 2018. Age-related changes in the neural networks supporting semantic cognition: A meta-analysis of 47 functional neuroimaging studies. Neuroscience \& Biobehavioral Reviews. 84:134-150. doi:10.1016/j.neubiorev.2017.11.010.

Jackson RL. 2021. The neural correlates of semantic control revisited. NeuroImage. 224:117444. doi:10.1016/j.neuroimage.2020.117444.

Jenkinson M, Beckmann CF, Behrens TEJ, Woolrich MW, Smith SM. 2012. FSL.

NeuroImage. 62(2):782-790. doi:10.1016/j.neuroimage.2011.09.015.

Kavé G, Knafo-Noam A. 2015. Lifespan development of phonemic and semantic fluency: Universal increase, differential decrease. Journal of Clinical and Experimental Neuropsychology. 37(7):751-763. doi:10.1080/13803395.2015.1065958.

Krieger-Redwood K, Jefferies E, Karapanagiotidis T, Seymour R, Nunes A, Ang JWA, Majernikova V, Mollo G, Smallwood J. 2016. Down but not out in posterior cingulate 
cortex: Deactivation yet functional coupling with prefrontal cortex during demanding semantic cognition. NeuroImage. 141:366-377. doi:10.1016/j.neuroimage.2016.07.060.

Krieger-Redwood K, Wang H-T, Poerio G, Martinon LM, Riby LM, Smallwood J, Jefferies E. 2019. Reduced semantic control in older adults is linked to intrinsic DMN connectivity. Neuropsychologia. 132:107133. doi:10.1016/j.neuropsychologia.2019.107133.

Law R, O’Carroll RE. 1998. A comparison of three measures of estimating premorbid intellectual level in dementia of the Alzheimer type. Int J Geriatr Psychiatry. 13(10):727730. doi:10.1002/(sici)1099-1166(1998100)13:10<727::aid-gps851>3.0.co;2-2.

Leech R, Braga R, Sharp DJ. 2012. Echoes of the Brain within the Posterior Cingulate Cortex. J Neurosci. 32(1):215-222. doi:10.1523/JNEUROSCI.3689-11.2012.

Lenth R. 2020. emmeans: Estimated Marginal Means, aka Least-Squares Means. R package version 1.4.8. https://CRAN.R-project.org/package=emmeans.

Li H-J, Hou X-H, Liu H-H, Yue C-L, Lu G-M, Zuo X-N. 2015. Putting age-related task activation into large-scale brain networks: A meta-analysis of 114 fMRI studies on healthy aging. Neuroscience \& Biobehavioral Reviews. 57:156-174. doi:10.1016/j.neubiorev.2015.08.013.

Li S-C, Lindenberger U, Sikström S. 2001. Aging cognition: from neuromodulation to representation. Trends in Cognitive Sciences. 5(11):479-486. doi:10.1016/S1364$\underline{6613(00) 01769-1 .}$

Lövdén M, Bäckman L, Lindenberger U, Schaefer S, Schmiedek F. 2010. A theoretical framework for the study of adult cognitive plasticity. Psychological Bulletin. 136(4):659676. doi:10.1037/a0020080.

Lüdecke D. 2018. ggeffects: Tidy Data Frames of Marginal Effects from Regression Models. JOSS. 3(26):772. doi:10.21105/joss.00772. 
Lurito JT, Kareken DA, Lowe MJ, Chen SHA, Mathews VP. 2000. Comparison of rhyming and word generation with FMRI. Human Brain Mapping. 10(3):99-106. doi:https://doi.org/10.1002/1097-0193(200007)10:3<99::AID-HBM10>3.0.CO;2-Q.

Mannhaupt, Hans-Rainer. 1983. Produktionsnormen für verbale Reaktionen zu 40 geläufigen Kategorien. Sprache \& Kognition.

Marsolais Y, Perlbarg V, Benali H, Joanette Y. 2014. Age-related changes in functional network connectivity associated with high levels of verbal fluency performance. Cortex. 58:123-138. doi:10.1016/j.cortex.2014.05.007.

McLaren DG, Ries ML, Xu G, Johnson SC. 2012. A Generalized Form of ContextDependent Psychophysiological Interactions (gPPI): A Comparison to Standard Approaches. Neuroimage. 61(4):1277-1286. doi:10.1016/j.neuroimage.2012.03.068.

Meinzer M, Flaisch T, Seeds L, Harnish S, Antonenko D, Witte V, Lindenberg R, Crosson B. 2012. Same Modulation but Different Starting Points: Performance Modulates Age Differences in Inferior Frontal Cortex Activity during Word-Retrieval. Stamatakis EA, editor. PLoS ONE. 7(3):e33631. doi:10.1371/journal.pone.0033631.

Meinzer M, Flaisch T, Wilser L, Eulitz C, Rockstroh B, Conway T, Gonzalez-Rothi L, Crosson B. 2009. Neural Signatures of Semantic and Phonemic Fluency in Young and Old Adults. Journal of Cognitive Neuroscience. 21(10):2007-2018. doi:10.1162/jocn.2009.21219.

Meinzer M, Seeds L, Flaisch T, Harnish S, Cohen ML, McGregor K, Conway T, Benjamin M, Crosson B. 2012. Impact of changed positive and negative task-related brain activity on word-retrieval in aging. Neurobiology of Aging. 33(4):656-669. doi:10.1016/j.neurobiolaging.2010.06.020. 
Morcom AM, Henson RNA. 2018. Increased Prefrontal Activity with Aging Reflects

Nonspecific Neural Responses Rather than Compensation. J Neurosci. 38(33):7303-7313. doi:10.1523/JNEUROSCI.1701-17.2018.

Morcom AM, Johnson W. 2015. Neural Reorganization and Compensation in Aging. Journal of Cognitive Neuroscience. 27(7):1275-1285. doi:10.1162/jocn_a_00783.

Nagels A, Kircher T, Dietsche B, Backes H, Marquetand J, Krug A. 2012. Neural processing of overt word generation in healthy individuals: The effect of age and word knowledge. NeuroImage. 61(4):832-840. doi:10.1016/j.neuroimage.2012.04.019.

Ng KK, Lo JC, Lim JKW, Chee MWL, Zhou J. 2016. Reduced functional segregation between the default mode network and the executive control network in healthy older adults: A longitudinal study. NeuroImage. 133:321-330. doi:10.1016/j.neuroimage.2016.03.029.

Noonan KA, Jefferies E, Visser M, Lambon Ralph MA. 2013. Going beyond Inferior Prefrontal Involvement in Semantic Control: Evidence for the Additional Contribution of Dorsal Angular Gyrus and Posterior Middle Temporal Cortex. Journal of Cognitive Neuroscience. 25(11):1824-1850. doi:10.1162/jocn_a_00442.

Noppeney U, Price CJ, Penny WD, Friston KJ. 2006. Two Distinct Neural Mechanisms for Category-selective Responses. Cereb Cortex. 16(3):437-445. doi:10.1093/cercor/bhi123. Nyberg L, Andersson M, Kauppi K, Lundquist A, Persson J, Pudas S, Nilsson L-G. 2014. Age-related and Genetic Modulation of Frontal Cortex Efficiency. Journal of Cognitive Neuroscience. 26(4):746-754. doi:10.1162/jocn_a 00521.

Oldfield RC. 1971. The assessment and analysis of handedness: the Edinburgh inventory. Neuropsychologia. 9(1):97-113. doi:10.1016/0028-3932(71)90067-4.

Park DC, Polk TA, Park R, Minear M, Savage A, Smith MR, Smith EE. 2004. Aging Reduces Neural Specialization in Ventral Visual Cortex. Proceedings of the National 
Academy of Sciences of the United States of America. 101(35):13091-13095.

doi:https://doi.org/10.1073/pnas.0405148101.

Persson J, Lustig C, Nelson JK, Reuter-Lorenz PA. 2007. Age Differences in Deactivation: A Link to Cognitive Control? Journal of Cognitive Neuroscience. 19(6):1021-1032. doi:10.1162/jocn.2007.19.6.1021.

Petrovich Brennan NM, Whalen S, de Morales Branco D, O’Shea JP, Norton IH, Golby AJ. 2007. Object naming is a more sensitive measure of speech localization than number counting: Converging evidence from direct cortical stimulation and fMRI. NeuroImage. 37:S100-S108. doi:10.1016/j.neuroimage.2007.04.052.

Pongpipat E, Kennedy K, Foster C, Boylan M, Rodrigue K. 2020 Dec 14. Functional connectivity within and between n-back modulated regions: An adult lifespan PPI investigation. Brain Connectivity. doi:10.1089/brain.2020.0791.

Poser BA, Versluis MJ, Hoogduin JM, Norris DG. 2006. BOLD contrast sensitivity enhancement and artifact reduction with multiecho EPI: parallel-acquired inhomogeneitydesensitized fMRI. Magn Reson Med. 55(6):1227-1235. doi:10.1002/mrm.20900.

R Core Team. 2018. R: A language and environment for statistical computing. Vienna, Austria: R Foundation for Statistical Computing. http://www.r-project.org/.

Reitan RM. 1958. Validity of the Trail Making Test as an indicator of organic brain damage. PMS. 8(7):271. doi:10.2466/PMS.8.7.271-276.

Schmidt CSM, Schumacher LV, Römer P, Leonhart R, Beume L, Martin M, Dressing A, Weiller C, Kaller CP. 2017. Are semantic and phonological fluency based on the same or distinct sets of cognitive processes? Insights from factor analyses in healthy adults and stroke patients. Neuropsychologia. 99:148-155. doi:10.1016/j.neuropsychologia.2017.02.019.

Schmidt K-H, Metzler P. 1992. WST-Wortschatztest. Göttingen: Beltz Test. 
Shao Z. 2014. What do verbal fluency tasks measure? Predictors of verbal fluency performance in older adults. Frontiers in Psychology.:10.

Shine JM, Bissett PG, Bell PT, Koyejo O, Balsters JH, Gorgolewski KJ, Moodie CA, Poldrack RA. 2016. The Dynamics of Functional Brain Networks: Integrated Network States during Cognitive Task Performance. Neuron. 92(2):544-554. doi:10.1016/j.neuron.2016.09.018.

Sidtis D, Canterucci G, Katsnelson D. 2009. Effects of neurological damage on production of formulaic language. Clinical Linguistics \& Phonetics. 23(4):270-284. doi: $10.1080 / 02699200802673242$.

Siegel JS, Power JD, Dubis JW, Vogel AC, Church JA, Schlaggar BL, Petersen SE. 2014. Statistical improvements in functional magnetic resonance imaging analyses produced by censoring high-motion data points. Hum Brain Mapp. 35(5):1981-1996. doi: $10.1002 / \mathrm{hbm} .22307$.

Singmann H, Bolker B, Westfall J, Aust F, Ben-Schachar MS. 2020. afex: Analysis of Factorial Experiments. R package version 0.27-2. https://CRAN.Rproject.org $/$ package $=$ afex.

Smith DV, Gseir M, Speer ME, Delgado MR. 2016. Toward a cumulative science of functional integration: A meta-analysis of psychophysiological interactions. Human Brain Mapping. 37(8):2904-2917. doi:10.1002/hbm.23216.

Spreng RN, DuPre E, Selarka D, Garcia J, Gojkovic S, Mildner J, Luh W-M, Turner GR. 2014. Goal-Congruent Default Network Activity Facilitates Cognitive Control. Journal of Neuroscience. 34(42):14108-14114. doi:10.1523/JNEUROSCI.2815-14.2014.

Spreng RN, Stevens WD, Viviano JD, Schacter DL. 2016. Attenuated anticorrelation between the default and dorsal attention networks with aging: evidence from task and rest. Neurobiology of Aging. 45:149-160. doi:10.1016/j.neurobiolaging.2016.05.020. 
Spreng RN, Turner GR. 2019. The Shifting Architecture of Cognition and Brain Function in Older Adulthood. :20.

Thompson-Schill SL, D’Esposito M, Aguirre GK, Farah MJ. 1997. Role of left inferior prefrontal cortex in retrieval of semantic knowledge: A reevaluation. PNAS. 94(26):14792-14797. doi:10.1073/pnas.94.26.14792.

Trelle AN, Henson RN, Simons JS. 2019. Neural evidence for age-related differences in representational quality and strategic retrieval processes. Neurobiology of Aging. 84:5060. doi:10.1016/j.neurobiolaging.2019.07.012.

Troyer A, Moscovitch M, Winocur G. 1997. Clustering and Switching as Two Components of Verbal Fluency: Evidence From Younger and Older Healthy Adults. Neuropsychology. 11(1):138-146. doi:10.1037//0894-4105.11.1.138.

Turner GR, Spreng RN. 2015. Prefrontal Engagement and Reduced Default Network Suppression Co-occur and Are Dynamically Coupled in Older Adults: The DefaultExecutive Coupling Hypothesis of Aging. Journal of Cognitive Neuroscience. 27(12):2462-2476. doi:10.1162/jocn_a_00869.

Tustison NJ, Avants BB, Cook PA, Zheng Y, Egan A, Yushkevich PA, Gee JC. 2010. N4ITK: improved N3 bias correction. IEEE Trans Med Imaging. 29(6):1310-1320. doi:10.1109/TMI.2010.2046908.

Vanlancker-Sidtis D, McIntosh AR, Grafton S. 2003. PET activation studies comparing two speech tasks widely used in surgical mapping. Brain and Language. 85(2):245-261. doi:10.1016/S0093-934X(02)00596-5.

Vatansever D, Menon DK, Manktelow AE, Sahakian BJ, Stamatakis EA. 2015. Default Mode Dynamics for Global Functional Integration. J Neurosci. 35(46):15254-15262. doi:10.1523/JNEUROSCI.2135-15.2015. 
Verhaegen P, Borchelt M, Smith J. 2003. Relation Between Cardiovascular and Metabolic Disease and Cognition in Very Old Age: Cross-Sectional and Longitudinal Findings From the Berlin Aging Study. Health Psychology. 22(6):559-569. doi:10.1037/02786133.22.6.559.

Vitali P, Abutalebi J, Tettamanti M, Rowe J, Scifo P, Fazio F, Cappa SF, Perani D. 2005. Generating animal and tool names: An fMRI study of effective connectivity. Brain and Language. 93(1):32-45. doi: 10.1016/j.band1.2004.08.005.

Wagner S, Sebastian A, Lieb K, Tüscher O, Tadić A. 2014. A coordinate-based ALE functional MRI meta-analysis of brain activation during verbal fluency tasks in healthy control subjects. BMC Neuroscience. 15(1):19. doi:10.1186/1471-2202-15-19.

Wechsler D. 1944. The measurement of adult intelligence (3rd ed.). Baltimore: Williams \& Wilkins Co. [accessed 2020 Oct 19]. http://content.apa.org/books/11329-000.

Whiteside DM, Kealey T, Semla M, Luu H, Rice L, Basso MR, Roper B. 2016. Verbal Fluency: Language or Executive Function Measure? Applied Neuropsychology: Adult. 23(1):29-34. doi:10.1080/23279095.2015.1004574.

Whitney C, Weis S, Krings T, Huber W, Grossman M, Kircher T. 2009. Task-dependent Modulations of Prefrontal and Hippocampal Activity during Intrinsic Word Production. Journal of Cognitive Neuroscience. 21(4):697-712. doi:10.1162/jocn.2009.21056.

Wickham H. 2016. ggplot2. Elegant Graphics for Data Analysis. Cham: Springer International Publishing (Use R!). [accessed 2020 Oct 19]. http://link.springer.com/10.1007/978-3-319-24277-4.

Wirth M, Jann K, Dierks T, Federspiel A, Wiest R, Horn H. 2011. Semantic memory involvement in the default mode network: A functional neuroimaging study using independent component analysis. NeuroImage. 54(4):3057-3066. doi:10.1016/j.neuroimage.2010.10.039. 
Xia M, Wang J, He Y. 2013. BrainNet Viewer: A Network Visualization Tool for Human Brain Connectomics. PLOS ONE. 8(7):e68910. doi:10.1371/journal.pone.0068910.

Zacks RT, Hasher L, Li KZH. 2000. Human memory. In: The handbook of aging and cognition, 2nd ed. Mahwah, NJ, US: Lawrence Erlbaum Associates Publishers. p. 293357.

Zonneveld HI, Pruim RHR, Bos D, Vrooman HA, Muetzel RL, Hofman A, Rombouts SARB, van der Lugt A, Niessen WJ, Ikram MA, et al. 2019. Patterns of functional connectivity in an aging population: The Rotterdam Study. NeuroImage. 189:432-444. doi:10.1016/j.neuroimage.2019.01.041. 